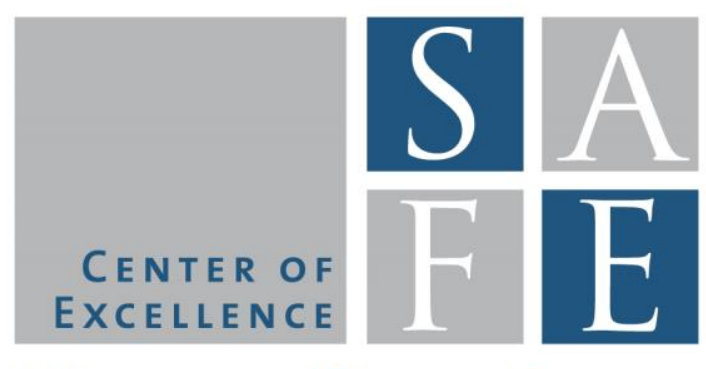

WORKINg PAPER SERIES

Marius Ascheberg - Nicole Branger - Holger Kraft - Frank Thomas Seifried

\title{
When Do Jumps Matter for Portfolio Optimization?
}

SAFE Working Paper Series No. 16

Center of Excellence SAFE Sustainable Architecture for Finance in Europe A cooperation of the Center for Financial Studies and Goethe University Frankfurt 


\title{
When Do Jumps Matter for Portfolio Optimization?
}

Marius Ascheberg ${ }^{\mathrm{a}}$, Nicole Branger ${ }^{\mathrm{b}}$, Holger Kraft, ${ }^{\text {, }}$, Frank Thomas Seifried ${ }^{\mathrm{d}}$

${ }^{a, c}$ Goethe University Frankfurt, Faculty of Economics and Business Administration, TheodorW.-Adorno-Platz 3, 60323 Frankfurt am Main, Germany.

${ }^{b}$ University of Muenster, Finance Center Muenster, Universitaetsstrasse 14-16, 48143 Muenster, Germany.

${ }^{d}$ Department IV - Mathematics, Universitaet Trier, Universitaetsring 19, 54296 Trier, Germany.

This version: November 25, 2015

\begin{abstract}
We consider the continuous-time portfolio optimization problem of an investor with constant relative risk aversion who maximizes expected utility of terminal wealth. The risky asset follows a jump-diffusion model with a diffusion state variable. We propose an approximation method that replaces the jumps by a diffusion and solve the resulting problem analytically. Furthermore, we provide explicit bounds on the true optimal strategy and the relative wealth equivalent loss that do not rely on quantities known only in the true model. We apply our method to a calibrated affine model. Our findings are threefold: Jumps matter more, i.e. our approximation is less accurate, if (i) the expected jump size or (ii) the jump intensity is large. Fixing the average impact of jumps, we find that (iii) rare, but severe jumps matter more than frequent, but small jumps.
\end{abstract}

JEL-Classification: G11; C63

Keywords: Optimal investment, jumps, stochastic volatility, welfare loss

* Corresponding author. Phone: +49 69798 33700; fax: +49 6979833901.

E-mail addresses: Marius.Ascheberg@hof.uni-frankfurt.de (Marius Ascheberg), Nicole.Branger@wiwi.uni-muenster.de (Nicole Branger), holgerkraft@finance.uni-frankfurt.de (Holger Kraft), seifried@uni-trier.de (Frank Thomas Seifried) 


\title{
When Do Jumps Matter for Portfolio Optimization?
}

This version: November 25, 2015

\begin{abstract}
We consider the continuous-time portfolio optimization problem of an investor with constant relative risk aversion who maximizes expected utility of terminal wealth. The risky asset follows a jump-diffusion model with a diffusion state variable. We propose an approximation method that replaces the jumps by a diffusion and solve the resulting problem analytically. Furthermore, we provide explicit bounds on the true optimal strategy and the relative wealth equivalent loss that do not rely on quantities known only in the true model. We apply our method to a calibrated affine model. Our findings are threefold: Jumps matter more, i.e. our approximation is less accurate, if (i) the expected jump size or (ii) the jump intensity is large. Fixing the average impact of jumps, we find that (iii) rare, but severe jumps matter more than frequent, but small jumps.
\end{abstract}

JEL-Classification: G11; C63

Keywords: Optimal investment, jumps, stochastic volatility, welfare loss 


\section{Introduction}

Jumps in stock prices or indices have an impact on the mean and variance of returns, but also on their higher moments. They usually increase the kurtosis and add positive or negative skewness to the distribution. Furthermore, they lead to discontinuous changes in portfolio values and are in this sense similar to illiquidity which prevents the investor from trading out of a position before too large losses are realized (see Liu, Longstaff, and Pan (2003)).

To study the relevance of jumps in a portfolio-choice context, we consider the continuous-time portfolio optimization problem of an investor who is exposed to jump risk and maximizes the expected utility of terminal wealth. The investor has access to a risky and a risk-free asset. ${ }^{1}$ The risky asset follows a generalized jump-diffusion process. Following a large strand of literature, ${ }^{2}$ we assume that the stock price dynamics involve the risk of downward jumps which capture unpredictable negative events. The state variable of the model follows a diffusion process. In general, this problem cannot be solved analytically, but one has to apply numerical methods to find the optimal portfolio strategy.

If the specific characteristics of jumps (in particular, their impact on higher moments) do not matter for portfolio optimization, then diffusion processes should approximate the effect of jumps on portfolio decisions reasonably well. We thus propose an approach to find approximating solutions for the optimal portfolio strategies that capture the effect of jumps via mean and variance only. Our approximation is based on a Taylor expansion of the jump-related terms in the Hamilton-Jacobi-Bellman (HJB) equation. This idea leads to an approximating portfolio problem in which the risky asset follows a diffusion process. The advantage of this approach is that the corresponding problem can be solved using a stochastic representation result. We refer to the resulting closed-form optimal strategies as approximating strategies. The crucial question arises how well these approximating strategies perform. More precisely, we address the question of to what extent the specific characteristics of jumps influence the

\footnotetext{
${ }^{1}$ This problem has been introduced in Merton (1969) and Merton (1971).

${ }^{2}$ See, e.g., Bates (2000), Eraker, Johannes, and Polson (2003), and Liu, Longstaff, and Pan (2003).
} 
accuracy of the approximation. We first give bounds for the difference between the (unknown) true strategy and the approximating strategy. Second, we find bounds for the relative wealth equivalent loss (RWEL), which is the proportion of wealth at time 0 that an investor using the optimal strategy can sacrifice in order to have the same indirect utility as an investor using the approximating strategy. ${ }^{3}$ These bounds can be determined without knowing the solution of the true model.

We apply our general results to an affine model with stochastic volatility and jumps in the stock price. The stochastic volatility of the stock is modeled as in Heston (1993), and the stock price is subject to jumps with a possibly stochastic jump size. Bates (1996, 2000) studies this class of models in the context of option pricing. The jump size is assumed to be constant, beta-distributed, or log-normally distributed. The parameters are similar to the ones in Pan (2002) and Liu, Longstaff, and Pan (2003), who calibrate the model to S\&P 500 option data. In the benchmark case, jumps happen on average every 25 years with an average jump size of $-25 \%$. Jumps are thus rare, but severe events. We provide an explicit solution for the approximating problem and use the numerical procedure from Liu, Longstaff, and Pan (2003) to also solve the true problem. For risk aversion levels of $\gamma>7$, we find that the bounds on the optimal strategies are less than $18 \%$ apart from each other. We apply the numerical method from Liu, Longstaff, and Pan (2003) to calculate exact RWELs for a ten year horizon. For the baseline calibration of the jumps, our approximating strategy performs well: All RWELs are below 1.16\% if jumps are stochastic, and the RWELs are below $1 \%$ if the jump size is constant and $\gamma \geq 5$.

To address the question of when jumps matter for portfolio optimization, we vary the jump characteristics and parametrizations. We find that jumps matter more, i.e. our approximation is less accurate, if the expected jump size or the jump intensity is large. Furthermore, we fix the average impact of jumps and show that rare, but severe jumps matter more than frequent, but small jumps. Finally, we study a second approximating strategy that is slightly

\footnotetext{
${ }^{3}$ This measure for the utility loss is e.g. also used by Liu, Longstaff, and Pan (2003) or Lynch (2001).
} 
more involved than the initial one, since a relevant constant is only implicitly given. Therefore, the strategy is not fully explicit. In our numerical examples, its performance is even better with RWELs of less than 5bps.

The remainder of the paper is organized as follows: Section 2 formulates a general model for a market with jumps in the stock price, presents our approximation procedure and derives bounds on the optimal strategy and on the RWEL resulting from this approximation. Section 3 considers an affine specification of the general model calibrated to S\&P 500 data. It provides

a numerical solution to the true problem and a closed-form solution to the approximating problem. Furthermore, we study the optimal and the approximating strategies in different settings and report the respective RWELs. The settings include varying risk aversions, jump sizes, and jump intensities. Section 4 concludes. Proofs that do not follow their statement can be found in the Appendix.

\section{Model and Portfolio Optimization}

We consider an economy where uncertainty is described by a complete filtered probability space denoted by $(\Omega, \mathcal{F}, \mathcal{P})$ where $\mathcal{F}=\left\{\mathcal{F}_{t}\right\}_{t \in[0, T]}$. The dynamics of the stock price $S$ are

$$
d S=S(\mu d t+\sigma d W-L d N)
$$

where $W$ is a Brownian motion and $N$ is a Cox process with intensity $\lambda$ and possibly stochastic jump size $L$. The drift $\mu$, the volatility $\sigma>0$, and the jump intensity $\lambda \geq 0$ are smooth functions of time $t$ and a state variable $Y$. The dynamics of $Y$ are given by

$$
d Y=\alpha d t+\beta d \tilde{W}
$$

Its drift $\alpha$ and volatility $\beta>0$ are also smooth functions of $t$ and $Y$, and $\tilde{W}$ is a correlated Brownian motion with $d W d \tilde{W}=\rho d t$. The correlation $\rho$ is constant. Unless stated otherwise, 
$Y$ is the local variance process of the stock, i.e. $\sigma^{2}=Y$.

\subsection{Portfolio Problem with Jumps}

We consider an investor who maximizes expected utility from terminal wealth with respect to a power utility function $u(x)=x^{1-\gamma} /(1-\gamma)$. He has constant relative risk aversion equal to $\gamma>1$, which is the empirically relevant case. ${ }^{4}$ His investment opportunities comprise a money market account with constant interest rate $r$ and a stock with dynamics given by (1). Hence, his wealth dynamics are given by

$$
d X=X[(r+\pi \chi) d t+\pi \sigma d W-\pi L d N]
$$

where $\pi$ denotes the proportion of wealth invested in stock, and $\chi \equiv \mu-r$. The stock's expected excess return is given by $\tilde{\chi} \equiv \mu-\bar{L}_{1} \lambda-r=\chi-\bar{L}_{1} \lambda$, where $\bar{L}_{k}$ denotes the $k$-th moment of $L$. The investor's indirect utility is $J(t, x, y) \equiv \sup _{\pi \in \mathcal{A}} \mathrm{E}^{t, x, y}\left[u\left(X_{T}\right)\right]$, where the supremum extends over admissible trading strategies and where $\mathrm{E}^{t, x, y}[\cdot]$ is shorthand for $\mathrm{E}\left[\cdot \mid X_{t}=x, Y_{t}=y\right]$. Here we focus on an appropriate class $\mathcal{A}$ of feedback (Markov) strategies of the form $\pi_{t}=\pi\left(t, X_{t}, Y_{t}\right){ }^{5}$ To avoid negative wealth, admissible strategies must satisfy

$$
1-\pi L \geq 0 \text { a.s. for all } \pi \in \mathcal{A}
$$

and we rule out short sales, i.e.

$$
\pi \geq 0 \quad \text { for all } \pi \in \mathcal{A}
$$

\footnotetext{
${ }^{4}$ See, e.g., Munk (2013) and the references therein.

${ }^{5}$ See Assumption 2.1 below. Given this assumption, the restriction to feedback strategies is without loss of generality.
} 
Remark. Since all relevant quantities are proportional to the investor's wealth, an elementary scaling argument implies that $J(t, x, y)=x^{1-\gamma} J(t, 1, y)$, so we have

$$
J(t, x, y)=\frac{1}{1-\gamma} x^{1-\gamma} g(t, y)
$$

for a suitable function $g$. In particular, it suffices to consider trading strategies $\pi_{t}=\pi\left(t, Y_{t}\right)$ that do not depend on the investor's current wealth $X_{t}$.

\subsection{HJB Equation}

In general, ${ }^{6}$ the indirect utility $J$ is a generalized solution (in the viscosity sense) of the associated Hamilton-Jacobi-Bellman (HJB) equation. For the portfolio problem described above, the HJB equation is given by

$$
\begin{gathered}
0=\sup _{\pi \geq 0,1-\pi L \geq 0 \text { a.s. }}\left\{J_{t}+x(r+\pi \chi) J_{x}+0.5 x^{2} \pi^{2} \sigma^{2} J_{x x}+\alpha J_{y}+0.5 \beta^{2} J_{y y}\right. \\
\left.+x \pi \sigma \beta \rho J_{x y}+\lambda[\mathrm{E}[J(t, x(1-\pi L), y)]-J(t, x, y)]\right\},
\end{gathered}
$$

where the expectation operator $\mathrm{E}[\cdot]$ represents the expectation over the jump size $L$ and $J(T, x, y)=u(x)$. In particular, if $J$ is sufficiently smooth, it is a classical solution of (6). We are not aware of conditions on the underlying coefficients of the portfolio problem that are necessary and sufficient for $J$ to be of class $C^{1,2,2}$, and a detailed analysis of this would be beyond the scope of this article. ${ }^{7}$ To focus on the issues of main interest here, we therefore impose the following technical condition throughout this article:

Assumption 2.1 There is a $C^{1,2,2}$ solution $J$ of the HJB equation (6) such that the local martingales $\int_{0}^{\cdot} \pi_{s} \sigma_{s} X_{s} J_{x}\left(s, X_{s}, Y_{s}\right) d W_{s}$ and $\int_{0}^{\cdot} \beta_{s} J_{y}\left(s, X_{s}, Y_{s}\right) d \tilde{W}_{s}$ are true martingales for every

\footnotetext{
${ }^{6}$ See, e.g., Alvarez and Tourin (1996), Zariphopoulou (2001), Barles and Imbert (2008) as well as Øksendal and Sulem (2005) and the references therein.

${ }^{7}$ In the absence of jumps, a set of general sufficient conditions can be found in Theorem 3.3 of Zariphopoulou (2001). In affine stochastic volatility models, Kallsen and Muhle-Karbe (2010) provide closed-form solutions.
} 
admissible strategy $\pi \in \mathcal{A}$, and the supremum in (6) is attained at an interior point $\pi^{*} \in \mathcal{A}$.

By a standard verification argument, Assumption 2.1 implies that the solution $J$ coincides with the investor's indirect utility function; that the maximizer $\pi^{*}$ represents the optimal trading strategy; and that $\pi^{*}$ satisfies the associated first-order condition.

One special case where it is straightforward to verify Assumption 2.1 is the affine model (22) of Section 3 with zero correlation, $\rho=0$. Then the optimal strategy $\pi^{*}$ is constant and the indirect utility function has an explicit affine representation (see Proposition 3.2). Under suitable requirements on $\chi$ and $L$ (e.g. $L$ attains its maximal value with positive probability) the constant $\pi^{*}$ is attained at an interior point.

Remark. Note that by (5) $J$ is automatically smooth with respect to $x$. Hence the validity of Assumption 2.1 depends on the function $g$, which is given in terms of a nonlinear partial integro-differential equation. ${ }^{8}$ Closed-form solutions to this equation are not available.

We wish to emphasize that, although this is not explicitly stated there, assumptions analogous to Assumption 2.1 (and Assumption 2.2 below) are made implicitly in a number of articles closely related to this one, including Kim and Omberg (1996), Liu, Longstaff, and Pan (2003), Liu (2007).

\subsection{Approximating Portfolio Problem}

To find an approximating solution to the portfolio problem, we use a formal second-order Taylor expansion for the jump term in the HJB equation (6):

$$
J(t, x(1-\pi L), y) \approx J(t, x, y)-J_{x}(t, x, y) x \pi L+0.5 J_{x x}(t, x, y)(x \pi L)^{2} .
$$

\footnotetext{
${ }^{8}$ See (35) in the Appendix.
} 
This implies $\mathrm{E}[J(t, x(1-\pi L), y)] \approx J(t, x, y)-J_{x}(t, x, y) x \pi \bar{L}_{1}+0.5 J_{x x}(t, x, y)(x \pi)^{2} \bar{L}_{2}$. Substituting this approximation into the HJB equation (6) yields

$$
\begin{gathered}
0=\sup _{\pi \geq 0}\left\{\tilde{J}_{t}+x\left[r+\pi(\underbrace{\chi-\lambda \bar{L}_{1}}_{\equiv \tilde{\chi}})\right] \tilde{J}_{x}+0.5 x^{2} \pi^{2}(\underbrace{\sigma^{2}+\lambda \bar{L}_{2}}_{\equiv \tilde{\sigma}^{2}}) \tilde{J}_{x x}\right. \\
\left.+\alpha \tilde{J}_{y}+0.5 \beta^{2} \tilde{J}_{y y}+x \pi \sigma \beta \rho \tilde{J}_{x y}\right\}
\end{gathered}
$$

subject to the boundary condition $J(T, x, y)=u(x)$. We continue to use $J$ to denote the solution of the HJB equation (6), while $\tilde{J}$ henceforth denotes the function in (8).

Note that (8) can be interpreted as the HJB equation that results from a model in which the jump-diffusion process for the stock price is replaced by a diffusion process with drift and volatility adjusted in such a way that the expected return and the local variance match those of the original process. For $L=0$, the two equations are of course identical. The main point is that the approximating portfolio problem can be solved in closed form. For problem (6), on the other hand, there is no closed-form solution available. In analogy to Assumption 2.1, we impose

Assumption 2.2 There exists a $C^{1,2,2}$ solution $\tilde{J}$ of the approximating HJB equation (8), and the supremum is attained at an interior point $\tilde{\pi}^{*}$.

It is now tempting to use $\tilde{\pi}^{*}$ as an "approximately optimal" strategy for the true portfolio problem. This, of course, raises the decisive question how good this approximation performs: How large is the utility loss from following the approximating strategy $\tilde{\pi}^{*}$ instead of the truly optimal strategy $\pi^{*}$ ? Furthermore, can we find an estimate for the approximation error solely by solving (8), i.e. without knowing the actual indirect utility $J$ and the actual optimal stock proportion $\pi^{*}$ ?

To address these points, we first solve the approximating problem (8). In the following, we assume that the jump intensity is proportional to the diffusive variance, i.e. there is a positive 
constant $K$ such that

$$
\lambda=K \sigma^{2} .
$$

This condition is for instance satisfied in the Heston model with jumps if we set $\lambda=K Y_{t}$.

Proposition 2.1 (Stochastic Representation of Approximated Indirect Utility) If condition (9) and suitable integrability conditions ${ }^{9}$ are satisfied, then the following hold:

(i) The indirect utility $\tilde{J}$ is given by

$$
\tilde{J}(t, x, y)=\frac{1}{1-\gamma} x^{1-\gamma} \tilde{f}(t, y)^{k} \quad \text { with } \quad k \equiv \frac{\gamma\left(1+K \bar{L}_{2}\right)}{\gamma\left(1-\rho^{2}+K \bar{L}_{2}\right)+\rho^{2}}=\text { const }
$$

where $\tilde{f}$ has the stochastic representation

$$
\tilde{f}(t, y)=\tilde{\mathrm{E}}^{t, y}\left[e^{-\int_{t}^{T} \tilde{r}_{u} d u}\right] \text { with }-\tilde{r} \equiv \frac{1-\gamma}{k}\left(r+0.5 \frac{1}{\gamma} \frac{\tilde{\chi}^{2}}{\tilde{\sigma}^{2}}\right)
$$

The expectation $\tilde{\mathrm{E}}[\cdot]$ is calculated using the measure $\tilde{\mathcal{P}}$ under which $Y$ has drift $\tilde{\alpha} \equiv \alpha+\frac{1-\gamma}{\gamma} \frac{\tilde{\chi} \sigma \beta \rho}{\tilde{\sigma}^{2}}$. (ii) If $(\tilde{\chi} / \tilde{\sigma})^{2}$ is increasing (decreasing) with respect to $Y$, then $\tilde{f}_{y}$ is negative (positive).

Notice that result (ii) can be used to determine the sign of the hedging term in the approximating strategy (see also the remark after Proposition 3.3).

In order not to drown the main ideas in technicalities, here and in the following we do not spell out detailed integrability and regularity conditions. The methodology developed in this article is rigorously justified whenever the standard tools of stochastic analysis (Feynman-Kac representation, differentiation under the integral, etc.) are applicable, which must be checked on a case-by-case basis.

\footnotetext{
${ }^{9} \mathrm{~A}$ sufficient condition is that all coefficients of the model are bounded with bounded derivatives of all orders, and that $\sigma$ and $\beta$ are bounded away from zero; see also Zariphopoulou (2001).
} 


\subsection{Bounds on Optimal Strategy}

In the following, we derive bounds on the optimal strategy $\pi^{*}$. We assume that the jump size distribution is positive:

$$
L \geq 0
$$

i.e. jumps only have a negative effect on the stock price. ${ }^{10}$ Furthermore, we do not allow for short sales.

Recall that the investor's indirect utility function is given by $J(t, x, y)=\frac{1}{1-\gamma} x^{1-\gamma} g(t, y)$. By Assumption 2.1, the optimal strategy $\pi^{*}=\pi$ is a solution of the first-order condition

$$
\gamma \sigma^{2} \pi=\chi+\sigma \beta \rho \frac{g_{y}}{g}-\lambda \mathrm{E}\left[L(1-\pi L)^{-\gamma}\right]
$$

To compare (13) with the first-order condition of the approximating HJB equation (8), we consider the first-order expansion $(1-\pi L)^{-\gamma}=1+\gamma \pi L+R_{1}(-\pi L)$, where

$$
R_{1}(-\pi L) \equiv \frac{\gamma(1+\gamma)}{2} \frac{1}{(1+\xi)^{\gamma+2}}(\pi L)^{2}
$$

denotes the remainder term of the expansion with a suitable intermediate value $\xi \in[-\pi L, 0]$. Therefore, the first-order condition (13) can be rewritten as

$$
\gamma(\underbrace{\sigma^{2}+\lambda \bar{L}_{2}}_{=\tilde{\sigma}^{2}}) \pi=\underbrace{\chi-\lambda \bar{L}_{1}}_{=\tilde{\chi}}+\sigma \beta \rho \frac{g_{y}}{g}-\lambda \mathrm{E}\left[L R_{1}(-\pi L)\right] .
$$

Condition (12) on the jump size and the short-sale constraint (4) together imply that the presence of jumps can never increase the investor's utility. Furthermore, by Assumption 2.1 we have $\pi^{*}>0$ and $1-\pi^{*} L>0$, so (12) guarantees that $\left|\pi^{*} L\right|<1$. This ensures in particular

\footnotetext{
${ }^{10}$ There is ample empirical evidence that jumps in stock index returns are on average negative. See, e.g., Eraker, Johannes, and Polson (2003) and Liu, Longstaff, and Pan (2003). We make the slightly more restrictive assumption (12) to rule out positive realized jumps. This helps to streamline the proofs and focus on our main points.
} 
that $\left(1-\pi^{*} L\right)^{-\gamma}$ has a well-defined binomial series expansion.

We first consider the case $\rho=0$. The first-order condition for the true optimal trading strategy $\pi^{*}$ becomes

$$
\pi^{*}=\frac{\tilde{\chi}}{\gamma \tilde{\sigma}^{2}}-\frac{\lambda \mathrm{E}\left[L R_{1}\left(-\pi^{*} L\right)\right]}{\gamma \tilde{\sigma}^{2}}
$$

The terms depending on $g$ have vanished. With zero correlation between the stock price and the state variable, the investor can no longer hedge changes in the investment opportunity set, and the hedging demand is zero. By abstracting from hedge terms, we can perform a clean analysis of the effect of jumps. As we will see later on, the general case is more involved. Given the results of Larsen and Munk (2012), who study sub-optimal strategies with misspecified hedge terms, this is not surprising. The following propositions give various bounds on the optimal strategy $\pi^{*}$.

Proposition 2.2 (Bounds on Optimal Stock Demand (I)) If (9) and (12) hold and $\rho=0$, then

$$
\tilde{\pi}^{*} \geq \pi^{*} \geq \frac{\tilde{\chi}}{\gamma \tilde{\sigma}^{2}}-\frac{(1+\gamma) \lambda}{2 \tilde{\sigma}^{2}}\left(\tilde{\pi}^{*}\right)^{2} C\left(\tilde{\pi}^{*}\right)
$$

where $C(\pi) \equiv \mathrm{E}\left[\frac{L^{3}}{(1-\pi L)^{\gamma+2}}\right]$. Besides, $\tilde{\pi}^{*}$ is adjusted to satisfy (3) if needed.

Proposition 2.2 uses the approximating strategy $\tilde{\pi}^{*}$ as an upper bound. The next proposition shows that we can find tighter upper and also lower bounds using a quadratic inequality.

Proposition 2.3 (Bounds on Optimal Stock Demand (II)) If (9) and (12) hold and $\rho=0$, then $\pi_{u} \geq \pi^{*} \geq \pi_{l}$, where for $j \in\{u, l\}$

$$
\pi_{j} \equiv-\frac{1}{2 A_{j}}+\sqrt{\frac{1}{\left(2 A_{j}\right)^{2}}+\frac{\tilde{\chi}}{\gamma \tilde{\sigma}^{2} A_{j}}}
$$

with $A_{u} \equiv \frac{(1+\gamma) \lambda}{2 \tilde{\sigma}^{2}} \bar{L}_{3}$ and $A_{l} \equiv \frac{(1+\gamma) \lambda}{2 \tilde{\sigma}^{2}} E\left[\frac{L^{3}}{\left(1-\pi_{u} L\right)^{\gamma+2}}\right]$. 
Next, we consider the general case $\rho \neq 0$. To derive bounds in this case, we further assume

$$
\rho g_{y} \geq 0
$$

which is satisfied for realistic calibrations of the Heston model. Without this condition, the Merton-Breeden term in the first-order condition (13) is negative and in general unbounded from below, which makes it very hard to derive bounds.

Proposition 2.4 (Bounds on Optimal Stock Demand (III)) If (9), (12) and (15) hold, then the optimal stock demand $\pi^{*}$ satisfies

$$
\bar{\pi} \geq \pi^{*} \geq \underline{\pi}
$$

where $\bar{\pi}$ is the optimal strategy in an auxiliary model where we set $L=0$, and $\underline{\pi}$ is the optimal strategy in an auxiliary model with $\rho=0$. The corresponding first-order conditions are

$$
\begin{aligned}
& \gamma \sigma^{2} \underline{\pi}=\chi-\lambda \mathrm{E}\left[L(1-\underline{\pi} L)^{-\gamma}\right], \text { and } \\
& \gamma \sigma^{2} \bar{\pi}=\chi+\sigma \beta \rho \frac{g_{y}^{n j}}{g^{n j}}
\end{aligned}
$$

where $g^{n j}$ denotes the relevant function in (5) for $L=0$ ("no jumps").

The following proposition can be used to verify condition (15). Here $\chi_{y},\left(\sigma^{2}\right)_{y}$, and $\pi_{y}^{*}$ denote the partial derivatives of $\chi, \sigma^{2}$, and $\pi^{*}$ with respect to $y$.

Proposition 2.5 (Sign of Hedge Term) Assuming (9) and sufficient regularity, the derivative $g_{y}$ is positive (negative) if

$$
(1-\gamma)\left[\pi_{y}^{*} \chi+\pi^{*} \chi_{y}-\gamma \pi^{*} \pi_{y}^{*} \sigma^{2}-0.5 \gamma \pi^{*}\left(\sigma^{2}\right)_{y}-\lambda \pi_{y}^{*} \mathrm{E}\left[L\left(1-\pi^{*} L\right)^{-\gamma}\right]+\lambda_{y}\left\{\mathrm{E}\left[\left(1-\pi^{*} L\right)^{1-\gamma}\right]-1\right\}\right.
$$


is positive (negative). If $\pi^{*}$ is deterministic, i.e. $\pi_{y}^{*}=0$, then (18) simplifies to

$$
(1-\gamma)\left[\pi^{*} \chi_{y}-0.5 \pi^{*}\left(\sigma^{2}\right)_{y}\right]+\lambda_{y}\left\{\mathrm{E}\left[\left(1-\pi^{*} L\right)^{1-\gamma}\right]-1\right\}
$$

\subsection{Bounds on Relative Wealth Equivalent Loss}

When the investor relies on the approximating strategy from solving (8), he suffers a utility loss. We measure this utility loss by the RWEL, that is the percentage of initial wealth that an investor using the optimal strategy can sacrifice and still have the same indirect utility as an investor using the approximating strategy. The investor's indirect utility in the original and the approximating model for two arbitrary feedback strategies $\pi, \tilde{\pi} \in \mathcal{A}$ are given by

$$
G(t, x, y ; \pi)=\frac{1}{1-\gamma} x^{1-\gamma} g(t, y ; \pi) \quad \text { and } \quad \tilde{G}(t, x, y ; \tilde{\pi})=\frac{1}{1-\gamma} x^{1-\gamma} \tilde{g}(t, y ; \tilde{\pi}) .
$$

The RWEL $\ell$ is defined implicitly by the identity

$$
\underbrace{G\left(t, x(1-\ell), y ; \pi^{*}\right)}_{=J(t, x(1-\ell), y)}=G\left(t, x, y ; \tilde{\pi}^{*}\right) .
$$

Using the functional form of $G$, the loss can be calculated as $\ell=1-\left(\frac{g\left(t, y ; \tilde{\pi}^{*}\right)}{g\left(t, y ; \pi^{*}\right)}\right)^{\frac{1}{1-\gamma}}$. To determine the exact loss, we thus need to know the truly optimal strategy $\pi^{*}$ and the utility $G$ in the original model. The challenge, however, is to find bounds for this loss that only depend on the optimal strategy $\tilde{\pi}^{*}$ and the utility $\tilde{G}$ in the approximating model.

Theorem 2.6 (Approximation Error) Suppose that $\pi, \tilde{\pi} \in \mathcal{A}$ are admissible strategies with $1-\pi L<1$, and assume sufficient integrability to apply the Feynman-Kac theorem.

(i) The difference of the indirect utilities in the original and approximating model reads

$$
G(t, x, y ; \pi)-\tilde{G}(t, x, y ; \tilde{\pi})=\frac{1}{1-\gamma} x^{1-\gamma} D(t, y ; \pi, \tilde{\pi})
$$


where $D(t, y ; \pi, \tilde{\pi}) \equiv g(t, y ; \pi)-\tilde{g}(t, y ; \tilde{\pi})=\int_{t}^{T} \hat{\mathrm{E}}^{t, y}\left[e^{-\int_{t}^{s} \hat{r}_{u} d u} \hat{D}_{s}\right] d s$ with $^{11}$

$$
\begin{aligned}
& \hat{D} \equiv\left\{\lambda \mathrm{E}\left[R_{2}(-\pi L)\right]-(1-\gamma) \Delta \pi\left[\chi-\lambda \bar{L}_{1}+\sigma \beta \rho \tilde{g}_{y} / \tilde{g}-0.5 \gamma(2+\Delta) \pi\left(\sigma^{2}+\lambda \bar{L}_{2}\right)\right]\right\} \tilde{g}, \\
& R_{2}(-\pi L) \equiv \frac{\gamma\left(1-\gamma^{2}\right)}{6} \frac{1}{(1+\xi)^{\gamma+2}}(-\pi L)^{3}, \quad \xi \in[-\pi L, 0], \\
&-\hat{r} \equiv(1-\gamma)\left(r+\pi \chi-0.5 \gamma \pi^{2} \sigma^{2}\right)+\lambda\left[\mathrm{E}\left[(1-\pi L)^{1-\gamma}\right]-1\right], \text { and } \hat{\alpha} \equiv \alpha+(1-\gamma) \pi \sigma \beta \rho . \quad \text { The }
\end{aligned}
$$
expectation $\hat{\mathrm{E}}[\cdot]$ is taken under a measure $\hat{\mathcal{P}}$ under which $Y$ has drift $\hat{\alpha}$ (instead of $\alpha$ ), and $\Delta$ is defined by $\tilde{\pi}=\pi(1+\Delta)$.

(ii) If $\tilde{\pi}=\tilde{\pi}^{*}$, then $\hat{D}$ simplifies into

$$
\hat{D}=\left\{\lambda E\left[R_{2}(-\pi L)\right]-0.5 \gamma(1-\gamma)(\pi \Delta)^{2}\left(\sigma^{2}+\lambda \bar{L}_{2}\right)\right\} \tilde{g}
$$

(iii) If condition (9) holds and $\tilde{\pi}=\tilde{\pi}^{*}$, then $\tilde{g}=\tilde{f}^{k}$ where $\tilde{f}$ and $k$ are given in (10).

Remarks. a) The representation of the utility difference $D$ does not depend on the unknown function $g$, but only on the function $\tilde{g}$ calculated in the approximating model.

b) The theorem is not restricted to the optimal strategies $\pi^{*}$ and $\tilde{\pi}^{*}$, but it holds for all admissible strategies $\pi, \tilde{\pi} \in \mathcal{A}$. It thus provides a general tool to find an estimate for the error the investor makes if he implements a misspecified strategy $\tilde{\pi}$ (instead of $\pi^{*}$ ). Of course, we are ultimately interested in the case where $\pi=\pi^{*}$ and $\tilde{\pi}=\tilde{\pi}^{*}$.

c) For parameterizations in which condition (12) holds, the remainder term $R_{2}$ is positive. Consequently, $\hat{D}$ and thus $D$ are positive if $\tilde{\pi}=\tilde{\pi}^{*}$. This implies $G \leq \tilde{G}$, i.e. the solution to the approximating problem overestimates the investor's indirect utility.

We now apply Theorem 2.6 to two specific situations, where the initial data $(t, y)$ are fixed:

$$
g\left(t, y ; \pi^{*}\right)=\tilde{g}\left(t, y ; \tilde{\pi}^{*}\right)+D\left(t, y ; \pi^{*}, \tilde{\pi}^{*}\right), \text { and } g\left(t, y ; \tilde{\pi}^{*}\right)=\tilde{g}\left(t, y ; \tilde{\pi}^{*}\right)+D\left(t, y ; \tilde{\pi}^{*}, \tilde{\pi}^{*}\right)
$$

\footnotetext{
${ }^{11}$ Here $\tilde{g}$ is short-hand for $\tilde{g}(\cdot, \cdot ; \tilde{\pi})$.
} 
$D\left(t, y ; \pi^{*}, \tilde{\pi}^{*}\right)$ represents the difference between the original model and the approximating model when we use the respective optimal strategies $\pi^{*}$ and $\tilde{\pi}^{*}$ in both models. $D\left(t, y ; \tilde{\pi}^{*}, \tilde{\pi}^{*}\right)$ gives the difference between the original model and the approximating model when we use the approximating optimal strategy $\tilde{\pi}^{*}$ in both models.

Theorem 2.6 and the bounds on the optimal strategy $\pi^{*}$ from Subsection 2.4 allow us to find bounds on these two functions. Suppressing their dependence on $(t, y)$ for simplicity, the bounds are denoted by

$$
\underline{D} \leq D\left(t, y ; \pi^{*}, \tilde{\pi}^{*}\right) \leq \bar{D}, \text { and } \underline{\tilde{D}} \leq D\left(t, y ; \tilde{\pi}^{*}, \tilde{\pi}^{*}\right) \leq \overline{\tilde{D}}
$$

This also gives bounds on $g\left(t, y ; \pi^{*}\right)$ and $g\left(t, y ; \tilde{\pi}^{*}\right)$ :

$\underline{D}+\tilde{g}\left(t, y ; \tilde{\pi}^{*}\right) \leq g\left(t, y ; \pi^{*}\right) \leq \bar{D}+\tilde{g}\left(t, y ; \tilde{\pi}^{*}\right), \quad$ and $\quad \underline{\tilde{D}}+\tilde{g}\left(t, y ; \tilde{\pi}^{*}\right) \leq g\left(t, y ; \tilde{\pi}^{*}\right) \leq \overline{\tilde{D}}+\tilde{g}\left(t, y ; \tilde{\pi}^{*}\right)$.

From these, we get lower and upper bounds for the ratio of the two functions:

$$
\underbrace{\frac{\tilde{D}+\tilde{g}\left(t, y ; \tilde{\pi}^{*}\right)}{\bar{D}+\tilde{g}\left(t, y ; \tilde{\pi}^{*}\right)}}_{\equiv \underline{B}} \leq \frac{g\left(t, y ; \tilde{\pi}^{*}\right)}{g\left(t, y ; \pi^{*}\right)} \leq \underbrace{\frac{\overline{\tilde{D}}+\tilde{g}\left(t, y ; \tilde{\pi}^{*}\right)}{D+\tilde{g}\left(t, y ; \tilde{\pi}^{*}\right)}}_{\equiv \bar{B}} .
$$

which then give upper and lower bounds on the RWEL: $1-(\underline{B})^{\frac{1}{1-\gamma}} \leq \ell \leq 1-(\bar{B})^{\frac{1}{1-\gamma}}$. Notice that $\underline{B}$ and $\bar{B}$ depend only on $\tilde{g}\left(t, y ; \tilde{\pi}^{*}\right)$ and on the bounds for $D$. In particular, they can be calculated without knowing the solution to the portfolio problem in the original model.

\section{An Affine Model}

We now consider a specific (affine) parametrization of the model that is given by

$$
\chi(t, y)=\bar{\chi} y, \quad \sigma(t, y)=\sqrt{y}, \quad \lambda(t, y)=\bar{\lambda} y, \quad \alpha(t, y)=\theta-\kappa y, \quad \beta(t, y)=\bar{\beta} \sqrt{y} .
$$


The state variable $Y$ is the local diffusion variance of the stock. It follows the square-root process from the model of Heston (1993). The stock price can jump, and with the jump intensity being proportional to the state variable $Y$, condition (9) is satisfied. This model setup is widely used in the option pricing literature, including Bates (1996) and Bates (2000). Liu, Longstaff, and Pan (2003) study the corresponding portfolio problem. They derive three equations that implicitly define the optimal investment strategy and indirect utility function, which have to be solved numerically. The following proposition summarizes their results.

Proposition 3.1 (Optimal Strategy in the Affine Model) In the model given by the affine specification (22), the investor's indirect utility is

$$
J(t, x, y)=\frac{1}{1-\gamma} x^{1-\gamma} e^{A(t)+B(t) y}
$$

where the following three equations implicitly define the optimal portfolio weight $\pi^{*}=\pi(t)$ and the functions $A$ and $B$ :

$$
\begin{aligned}
\bar{\chi}-\gamma \pi(t)+\bar{\beta} \rho B(t)-\bar{\lambda} \mathrm{E}\left[(1-\pi(t) L)^{-\gamma} L\right] & =0 \\
\left\{B_{t}(t)+B(t)[(1-\gamma) \bar{\beta} \rho \pi(t)-\kappa]+\bar{\lambda} \mathrm{E}\left[(1-\pi(t) L)^{1-\gamma}\right]\right. & \\
\left.+\frac{1}{2} \bar{\beta}^{2} B(t)^{2}+\left[\pi(t)^{2} \frac{\gamma(\gamma-1)}{2}+\pi(t)(1-\gamma) \bar{\chi}-\bar{\lambda}\right]\right\} & =0 \\
A_{t}(t)+(1-\gamma) r+\theta B(t) & =0
\end{aligned}
$$

The boundary conditions are $A(T)=B(T)=0$.

These equations can be solved numerically by applying a backward differencing scheme. Furthermore, if we use a (not necessarily optimal) strategy $\pi$, equations (25) and (26) allow us to calculate the associated expected utility.

When the correlation between the stock price and its volatility is zero, the optimal strategy and indirect utility become more explicit. 
Proposition 3.2 (Uncorrelated Local Variance) Consider the model (22) and suppose that $\rho=0$.

(i) The optimal investment policy $\pi^{*}$ is constant over time, independent of the local variance $Y$ and implicitly given by

$$
\gamma \pi^{*}=\bar{\chi}-\bar{\lambda} \mathrm{E}\left[L\left(1-\pi^{*} L\right)^{-\gamma}\right]
$$

(ii) The indirect utility for a given constant strategy $\pi$ is ${ }^{12}$

$$
G_{0}(t, x, y ; \pi)=\frac{1}{1-\gamma} x^{1-\gamma} g_{0}(t, y ; \pi), \text { with } g_{0}(t, y ; \pi) \equiv e^{(1-\gamma) r(T-t)+A_{0}(t, T)+B_{0}(t, T) y},
$$

where

$$
\begin{aligned}
A_{0}(t, T) & \equiv \frac{2 \theta}{\bar{\beta}^{2}} \ln \left(\frac{2 a e^{\frac{1}{2}(a+\kappa)(T-t)}}{2 a+(a+\kappa)\left(e^{a(T-t)}-1\right)}\right), \quad B_{0}(t, T) \equiv \frac{2 C_{0}\left(e^{a(T-t)}-1\right)}{2 a+(a+\kappa)\left(e^{a(T-t)}-1\right)}, \\
C_{0} & \equiv(1-\gamma)\left(\pi \bar{\chi}-\frac{1}{2} \gamma \pi^{2}+\frac{\bar{\lambda}}{1-\gamma}\left\{\mathrm{E}\left[(1-\pi L)^{1-\gamma}\right]-1\right\}\right),
\end{aligned}
$$

and $a \equiv \sqrt{\kappa^{2}-2 \bar{\beta}^{2} C_{0}}$. The indirect utility of the optimal strategy is thus $J_{0}(t, x, y)=G_{0}\left(t, x, y ; \pi^{*}\right)$, with $\pi^{*}$ implicitly defined by (27).

(iii) The approximating strategy $\tilde{\pi}^{*}$ is also constant and given by

$$
\tilde{\pi}^{*}=\frac{\bar{\chi}-\bar{\lambda} \bar{L}_{1}}{\gamma\left(1+\bar{\lambda} \bar{L}_{2}\right)}
$$

(iv) The RWEL is given in closed form by

$$
1-\left[\frac{g_{0}\left(t, y ; \tilde{\pi}^{*}\right)}{g_{0}\left(t, y ; \pi^{*}\right)}\right]^{\frac{1}{1-\gamma}}
$$

We now turn to the general case $\rho \neq 0$. In the approximating market, we have $\tilde{\alpha}=\theta-\tilde{\kappa} y$ with $\tilde{\kappa}=\kappa+\frac{\gamma-1}{\gamma} \frac{\tilde{\chi} \bar{\beta} \rho}{\overline{\bar{\sigma}}^{2}}$ where $\tilde{\bar{\chi}}=\bar{\chi}-\bar{\lambda} \bar{L}_{1}$ and $\tilde{\tilde{\sigma}}^{2}=1+\bar{\lambda} \bar{L}_{2}$. We apply Proposition 2.1 to

\footnotetext{
${ }^{12}$ We use the subscript 0 to indicate that we assume $\rho=0$.
} 
obtain an explicit solution of the approximating problem. ${ }^{13}$

Proposition 3.3 (Approximating Problem in the Affine Model) For the affine model (22), it holds that

$$
\tilde{f}(t, y)=e^{-\tilde{A}(t, T)-\tilde{B}(t, T) y}
$$

The functions $\tilde{A}$ and $\tilde{B}$ are

$$
\begin{aligned}
\tilde{B}(t, T) & \equiv 2 b \frac{e^{a(T-t)}-1}{e^{a(T-t)}(\tilde{\kappa}+a)-\tilde{\kappa}+a}, \text { and } \\
\tilde{A}(t, T) & \equiv \frac{2 \theta}{\bar{\beta}^{2}} \ln \left(\frac{1-q e^{-a(T-t)}}{1-q}\right)+\left(\frac{2 \theta b}{\tilde{\kappa}+a}-\frac{1-\gamma}{k} r\right)(T-t),
\end{aligned}
$$

where $a \equiv \sqrt{\tilde{\kappa}^{2}+2 b \bar{\beta}^{2}}, b \equiv 0.5 \frac{\gamma-1}{k \gamma} \tilde{\bar{\eta}}^{2}, \tilde{\bar{\eta}} \equiv \tilde{\bar{\chi}} / \tilde{\bar{\sigma}}, q=(\tilde{\kappa}-a) /(\tilde{\kappa}+a)$, and the constant $k$ is given in Equation (10) with $K \equiv \bar{\lambda}$.

Remark. Obviously, $\tilde{B} \geq 0$ and thus $\tilde{f}_{y}(t, y)=-\tilde{B}(t, T) \tilde{f}(t, y) \leq 0$. This also follows from Proposition 2.1 (ii) since $\frac{\tilde{\chi}^{2}}{\tilde{\sigma}^{2}}=\frac{\tilde{\chi}^{2}}{\tilde{\sigma}^{2}} y$ is increasing in $y$.

\subsection{Numerical Results}

We use the following parameters that are similar to the ones in Liu, Longstaff, and Pan $(2003):^{14}$

$$
\bar{\chi}=5.363, r=0.028, \theta=0.115, \kappa=5.30, \bar{\beta}=0.225, \rho=-0.57, \bar{\lambda}=1.842 .
$$

The mean-reversion level of the local variance $Y$ is $\theta / \kappa=0.0217$. Jumps are assumed to be rare, but severe events. The expected jump size is $25 \%$ and jumps arrive on average about every 25 years (the mean-reversion level of the jump intensity is $\bar{\lambda} \theta / \kappa=0.04$ ). The jump intensity is linear in the local variance such that the frequency of jumps increases in volatile

\footnotetext{
${ }^{13}$ See also Kallsen and Muhle-Karbe (2010).

${ }^{14}$ Note that the estimates of Liu, Longstaff, and Pan (2003) also include jumps in volatility.
} 
times. Empirical studies suggest that this is a feature observed in the data (see, e.g. Pan (2002)). We consider three different jump size distributions $L$ :

(i) A constant jump size $L=0.25$. The admissibility condition (3) implies $\pi<4$.

(ii) A beta-distributed jump size $L \sim K_{L} \mathcal{B}\left(\alpha_{L}, \beta_{L}\right)$. We set $\alpha_{L}=18.5, \beta_{L}=55.5$, and $K_{L}=1.0$. This implies an expected stock jump size of $\mathrm{E}[L]=0.25$ and a standard deviation of $\mathrm{SD}[L]=0.05$. The support of the jump size distribution is $\left[0, K_{L}\right]$. The admissibility condition (3) implies $\pi<\frac{1}{K_{L}}=1$.

(iii) A shifted log-normal jump size $L=1-e^{\mu_{L}+\sigma_{L} Z}$, where $Z$ is a standard normal random variable. We set $\mu_{L}=-0.2965$ and $\sigma_{L}=0.1327$, which implies $\mathrm{E}[L]=0.25$ and $\mathrm{SD}[L]=$ 0.10. The support of the jump size distribution is $(-\infty, 1]$, so $(3)$ implies $0<\pi<1$. Note that this jump size distribution violates condition (12), which is needed to calculate bounds on the optimal strategy. We still report the bounds even though the condition is not satisfied and check whether they give sensible results also in this case. ${ }^{15}$

\subsection{Optimal Strategies and Bounds for Zero Correlation}

We first consider the case $\rho=0$. The optimal strategy and the approximating strategy follow from Theorem 3.2. The bounds on the optimal strategy follow from Propositions 2.2 and 2.3. Figure 1 illustrates the optimal investment strategy $\pi^{*}$, the approximating investment strategy $\tilde{\pi}^{*}$ and the bounds on the optimal investment strategy for a constant jump size as a function of relative risk aversion $\gamma$. In line with intuition, the optimal and the approximating portfolio weight decrease in $\gamma$. This also holds true for the distance between the bounds and the optimal strategy $\pi^{*}$ as well as for the distance between the approximating strategy $\tilde{\pi}^{*}$ and the optimal strategy $\pi^{*}$. By construction, the bounds II are narrower than the bounds I. They particularly improve on the lower bound for low levels of risk aversion, for which the lower bound I is sometimes even negative. For risk aversion levels of $\gamma>7$, the difference between

\footnotetext{
${ }^{15}$ We have deliberately chosen a higher standard deviation than for the beta distribution to make positive jump realizations more likely. See also the discussion of Table 1 below.
} 
the upper and lower bound II is below 10\%. The difference between the approximating and optimal strategy is below $5 \%$ for $\gamma>7$.

Figure 2 depicts the corresponding results if the jump size is beta-distributed. The upper bound $\pi \leq 1$ which follows from the admissibility condition (3) is now actually binding for low levels of risk aversion. The lower and upper bounds are weaker than for a constant jump size. For $\gamma>7$, the upper and lower bounds II are less than $12 \%$ apart from each other. The approximating and optimal strategies differ by less than $6 \%$ for $\gamma>7$. Figure 3 depicts the same results for log-normal jumps. ${ }^{16}$ For $\gamma>7$, the upper and lower bounds II differ by less than $18 \%$. The approximating and optimal strategies are less than $8 \%$ apart for $\gamma>7$. To summarize, both the approximating strategy and the bounds are reasonable close to the optimal strategy.

\subsection{Bounds for Negative Correlation}

We now consider a model with $\rho=-0.57$ and analyze the bounds III implied by Proposition 2.4. We do not report the other bounds as they require $\rho=0$. The upper bound does not depend on the type of jumps and is thus identical for all three jump types. For risk aversion levels between 2 and 10 this upper bound is very large and is thus not reported. Figure 4 depicts the lower bounds and the corresponding optimal strategies for $t=0$. We observe little differences between the lower bounds for deterministic and stochastic jump sizes. The lower bounds for different stochastic jump size distributions are also very close to each other. The admissibility condition $(3)$ is binding for low risk aversion levels $(\gamma<4)$. To summarize, the bounds on the optimal strategy $\pi^{*}$ for $\rho=-0.57$ are not very tight, but the optimal strategies are rather close to the lower bounds.

\footnotetext{
${ }^{16}$ Notice that the results of Section 2.4 require $L \geq 0$.
} 


\subsection{Relative Wealth Equivalent Losses}

We now examine how the approximating strategy performs compared to the true optimal strategy. The comparison is done via the RWEL. This loss measure is independent of the current level of wealth, but depends on the local variance $Y$. In the following, we set the local variance equal to its mean reversion level. Furthermore, we assume a time horizon of $T=10$ years.

For a correlation of $\rho=0$, we can use Theorem 3.2 to calculate the RWEL explicitly. Figure 5 shows the RWEL for different assumptions about the jump size as a function of the relative risk aversion $\gamma$. For a constant jump size, the losses decrease monotonically in $\gamma$, and they are below $1 \%$ if $\gamma>4$. For beta-distributed and log-normally distributed jumps, the losses are below $1 \%$ and $2 \%$, respectively. In both cases, the upper bound from (3) is binding for the optimal and approximating strategies for small values of $\gamma$. The optimal and the approximating strategy then coincide, which of course brings the RWEL down to zero. When $\gamma$ increases and the restrictions are not binding anymore, RWELs first increase and then decrease again.

To calculate RWELs for the case $\rho=-0.57$, we can use the numerical procedure of Proposition 3.1. Table 1 reports the RWELs for risk aversion levels $\gamma$ between 2 and 10. For low risk aversion levels, there are high losses only when the jump size is constant, while the losses are zero for stochastic jump sizes. This is again due to condition (3) that implies an upper bound on the admissible strategies if the jump size is stochastic. This upper bound becomes binding if the relative risk aversion is small. As a result, the RWELs are all below 1.16\% if jumps are stochastic. They are below $1 \%$ if the jump size is constant and $\gamma \geq 5$. Notice that the losses are smaller for log-normally distributed jumps than for beta-distributed jumps, although the average loss is the same in both cases. This is also true in Tables 2-4 below.

Next, we study the dependence of RWELs on the expected stock jump size. In our model, the

expected excess return on the stock is $(\bar{\chi}-\mathrm{E}[L] \bar{\lambda}) y$. When we change the expected jump 
size $E[L]$, we offset its impact on the expected excess return by simultaneously changing $\bar{\chi}$. For constant jumps we simply set the jump size equal to the new expected jump size. For betadistributed jumps, we adjust the support of the jump size distribution. ${ }^{17}$ For log-normally distributed jump sizes, we only change the expected jump size and leave the volatility fixed at $10 \%$.

Table 2 reports the RWELs for different expected jump sizes ranging from 0.05 to 0.30 for a constant jump size, a beta-distributed jump size, and a log-normally distributed jump size, respectively. In line with intuition, higher expected jump sizes result in higher RWELs across all risk aversion levels. If the expected jump size is below 15\%, the RWELs are below 21bps (constant), 10bps (beta distribution), and 2bps (log-normal distribution). The highest RWELs are obtained for an expected jump size of 30\%: $14.38 \%$ (constant, $\gamma=2$ ), $2.55 \%$ (beta distribution, $\gamma=5$ ), and 2.03\% (log-normal distribution, $\gamma=5$ ). To summarize, our approximation performs well if the jump size is stochastic or if it is constant and below $20 \%$. Next, we study the dependence of RWELs on the jump intensity. As before, we fix the expected excess return on the stock and use $\bar{\chi}$ to offset the change in the jump intensity parameter $\bar{\lambda}$. Table 3 reports the RWELs for different jump intensity parameters $\bar{\lambda}$ ranging from 0.1 to 4.0 (the benchmark value is $\bar{\lambda}=1.84156$ ). ${ }^{18}$ Results for all three jump size specifications are reported. Larger jump intensities result in higher RWELs across all risk aversion levels. If $\gamma \geq 5$, the RWELs are below $2 \%$ for all jump types and $\bar{\lambda} \in[0.1,4.0]$. The highest RWELs are 8.41\% (constant, $\gamma=2.0, \bar{\lambda}=4.0$ ), 2.66\% (beta distribution, $\gamma=4.0$, $\bar{\lambda}=4.0$ ), and $1.83 \%$ (log-normal distribution, $\gamma=5.0, \bar{\lambda}=4.0)$.

Finally, we vary both jump size and jump intensity, but keep the product $\bar{\lambda} \bar{L}_{1}$ fixed. Therefore, we do not change the average impact of jumps and address the question whether our

\footnotetext{
${ }^{17}$ We set $K_{L}$ to be twice the expected stock jump size. Additionally, we linearly scale the volatility of the stock jump size with respect to the maximal stock jump size $K_{L}$ according to $\operatorname{Vol}(L)=0.01 \frac{K_{L}}{0.5}$. This procedure avoids unrealistic or even non well-defined distributions. If the volatility would be left at 0.1 for an expected jump size of 0.05 with a maximal jump size of 0.1 , we cannot obtain a fit with a beta distribution.

${ }^{18}$ Note that the resulting jump intensity is $\bar{\lambda} Y$. If $Y$ is equal to its mean reversion level, the jump intensity varies from $22 \mathrm{bps}$ to $8.69 \%$.
} 
approximate strategy performs better for rare, but severe jumps or frequent, but moderate jumps. Intuitively, we expect that utility losses are smaller for the latter case since Taylor approximations are in general more accurate around the expansion point. Table 4 confirms this intuition for all jump parametrizations. In every row, the losses are monotonically increasing in the expected jump size. These results suggest that jumps matter the most when they are rare, but severe.

\subsection{An Alternative Approximate Strategy}

Our previous results suggest that our approximating strategy performs well in most cases. Nevertheless, approximating the jump component by adjusting the diffusion components implies that a typical risk characteristic of jumps is lost. In particular, we ignore the illiquidity character of jumps, which is discussed in Liu, Longstaff, and Pan (2003).

We thus construct an alternative approximating strategy. For $\rho=0$, we solve for the optimal and approximating strategies $\pi_{0}^{*}$ and $\tilde{\pi}_{0}^{*}$ by using Theorem 3.2. Both strategies are constant over time. They ignore the impact of correlation, but the difference between them accounts for the characteristics of jumps. We now use this difference as a proxy for the missing component in the approximating strategy. If $\tilde{\pi}^{*}$ is the approximating strategy for general $\rho \neq 0$, we define the new approximating strategy by

$$
\tilde{\pi}_{2} \equiv \tilde{\pi}^{*}+\left(\pi_{0}^{*}-\tilde{\pi}_{0}^{*}\right)
$$

The strategy in (31) requires only the approximating strategy $\tilde{\pi}^{*}$, the approximating strategy $\tilde{\pi}_{0}^{*}$ for $\rho=0$, and the optimal strategy $\pi_{0}^{*}$ for $\rho=0$. The first two are known explicitly, the last is constant over time and can be computed from (27).

We calculate RWELs using the numerical procedure of Proposition 3.1. As before, we run robustness checks with respect to risk aversion, the expected jump size, and the jump intensity for all three jump types. Table 5 shows that the adjusted strategies perform very well. For 
the same parameter intervals as considered above, the largest RWEL is below 5 basis points. It is obtained for a small risk aversion of $\gamma=2$ and a large constant jump size of $30 \%$.

\section{Conclusion}

We consider the continuous-time portfolio optimization problem of an investor with constant relative risk aversion who maximizes expected utility of terminal wealth in a model in which the risky asset follows a generalized (not necessarily affine) jump-diffusion model with a stochastic state process. This problem cannot be solved analytically. We use a Taylor approximation to obtain an approximating portfolio problem and provide an analytical stochastic representation of its solution. Furthermore, we derive bounds on the deviation between the optimal and the approximating strategy and bounds on the RWELs. These bounds do not depend on the solution of the portfolio planning problem in the original market and can thus be calculated even when this solution is unknown.

Furthermore, we study a specific affine parametrization of the portfolio problem in which the risky asset follows a Heston (1993) process with jumps. We provide an explicit solution to the approximating problem and use the numerical procedure from Liu, Longstaff, and Pan (2003) to solve the true problem. It turns out that the utility losses resulting from approximating a jump by a diffusion process are negligible if expected jump sizes are below 20\%. One key finding is that jumps matter more, i.e. our approximation is less accurate, when jumps are rare, but severe events. By contrast, the effect of frequent, but moderate jumps on optimal portfolio choice is small.

\section{References}

Alvarez, Olivier, and Agnès Tourin, 1996, Viscosity Solutions of Nonlinear Integro-Differential Equations, Annales de l'Institut Henri Poincaré (C) 13, 293-317. 
Barles, Guy, and Cyril Imbert, 2008, Second-Order Elliptic Integro-Differential Equations: Viscosity Solutions' Theory Revisited, Annales de l'Institut Henri Poincaré (AN) 25, 567585.

Bates, David S., 1996, Jumps and Stochastic Volatility: Exchange Rate Processes Implicit in Deutsche Mark Options, Review of Financial Studies 9-1, 69-107.

Bates, David S., 2000, Post-'87 Crash Fears in the S\&P 500 Futures Option Market, Journal of Econometrics 94, 181-238.

Cont, Rama, and Peter Tankov, 2004, Financial Modelling with Jump Proceses. CRC Press.

Eraker, Bjørn, Michael Johannes, and Nicholas Polson, 2003, The Impact of Jump in Volatility and Returns, Journal of Finance LVIII.

Friedman, Avner, 1975, Stochastic Differential Equations and Applications. Academic Press.

Heston, Steven L., 1993, A Closed-Form Solution for Options with Stochastic Volatility with Applications to Bond and Currency Options, Review of Financial Studies 6-2, 327-343.

Kallsen, Jan, and Johannes Muhle-Karbe, 2010, Utility Maximization in Affine Stochastic Volatility Models, International Journal of Theoretical and Applied Finance 13, 459-477.

Kim, Tong Suk, and Edward Omberg, 1996, Dynamic Nonmyopic Portfolio Behavior, Review of Financial Studies 9-1, 141-161.

Larsen, Linda S., and Claus Munk, 2012, The Costs of Suboptimal Dynamic Asset Allocation: General Results and Applications to Interest Rate Risk, Stock Volatility Risk, and Growth/Value Tilts, Journal of Economic Dynamics and Control 36-2, 266-293.

Liu, Jun, 2007, Portfolio Selection in Stochastic Environments, Review of Financial Studies $20,1-39$. 
Liu, Jun, Francis A. Longstaff, and Jun Pan, 2003, Dynamic Asset Allocation with Event Risk, Journal of Finance 58, 231-260.

Lynch, Anthony W, 2001, Portfolio choice and equity characteristics: Characterizing the hedging demands induced by return predictability, Journal of Financial Economics 62, 67-130.

Merton, Robert C., 1969, Lifetime Portfolio Selection under Uncertainty: The ContinuousTime Case, Review of Economics and Statistics 51-3, 247-257.

Merton, Robert C., 1971, Optimum Consumption and Portfolio Rules in a Continuous-Time Model, Journal of Economic Theory 3, 373-413.

Munk, Claus, 2013, Financial Asset Pricing Theory. Oxford University Press, Oxford.

Øksendal, Bernt, and Agnès Sulem, 2005, Applied Stochastic Control of Jump Diffusions. Springer.

Pan, Jun, 2002, The Jump-risk Premia Implicit in Options: Evidence from an Integrated Time-series Study, Journal of Financial Economics 63, 3-50.

Protter, Philip E., 2005, Stochastic Integration and Differential Equations. Springer, second edition.

Zariphopoulou, Thaleia, 2001, A Solution Approach to Valuation with Unhedgeable Risks, Finance and Stochastics 5, 61-82. 


\section{A Proofs}

Proof of Proposition 2.1. (i) Using (5) we write $\tilde{J}(t, x, y)=\frac{1}{1-\gamma} x^{1-\gamma} \tilde{f}(t, y)^{k}, \tilde{f}(T, y)=1$ with $k$ chosen as in (10). Substituting into (8) yields $\tilde{\pi}^{*}=\frac{\tilde{\chi}}{\gamma \tilde{\sigma}^{2}}+\frac{k \sigma \beta \rho}{\gamma \tilde{\sigma}^{2}} \frac{\tilde{f}_{y}}{\tilde{f}}$, where

$$
0=\tilde{f}_{t}+\underbrace{\frac{1-\gamma}{k}\left(r+0.5 \frac{1}{\gamma} \frac{\tilde{\chi}^{2}}{\tilde{\sigma}^{2}}\right)}_{\equiv-\tilde{r}} \tilde{f}+\underbrace{\left(\alpha+\frac{1-\gamma}{\gamma} \frac{\tilde{\chi} \sigma \beta \rho}{\tilde{\sigma}^{2}}\right)}_{\tilde{\alpha}} \tilde{f}_{y}+0.5 \beta^{2} \tilde{f}_{y y} .
$$

Applying Feynman-Kac to (32) gives the representation (11) of $\tilde{f}$. Note that, if all coefficients are bounded and $\sigma$ and $\beta$ are bounded away from zero, then (32) is elliptic with bounded coefficients, so the conditions of the Feynman-Kac theorem are satisfied. ${ }^{19}$

(ii) We set $\tilde{R}\left(t, Y_{t}\right) \equiv \tilde{r}_{t}$. $\tilde{R}_{y}=\partial \tilde{R} / \partial y$ denotes the partial derivative of the function $\tilde{R}$ with respect to its second argument. Then, using appropriate integrability conditions on the underlying coefficients that ensure differentiability of $\tilde{f}, \tilde{f}_{y}(t, y)=\tilde{\mathrm{E}}^{t, y}\left[e^{-\int_{t}^{T} \tilde{r}_{u} d u} \int_{t}^{T}-\tilde{R}_{y}\left(u, Y_{u}\right) P_{u} d u\right]$, where $P_{u} \equiv \frac{\partial}{\partial y} Y_{u}$ denotes the derivative of the process $Y$ with respect to its initial value $Y(t)=$ $y \cdot{ }^{20} P$ satisfies the stochastic differential equation $d P_{s}=P_{s}\left[\tilde{\alpha}_{y}\left(s, Y_{s}\right) d t+\beta_{y}\left(s, Y_{s}\right) d \widetilde{\widetilde{W}}\right]$, where $\widetilde{\widetilde{W}}$ is a $\tilde{\mathcal{P}}$-Brownian motion. Since $P \geq 0$, the sign of $f_{y}$ is negative if $-\tilde{R}_{y}=\frac{1-\gamma}{2 k \gamma} \frac{\partial(\tilde{\chi} / \tilde{\sigma})^{2}}{\partial y} \leq 0$ and positive if $-\tilde{R}_{y} \geq 0$. Since $\gamma>1$ by assumption, the claim follows.

Proof of Proposition 2.2. Our assumption yields $0 \leq \pi^{*} L \leq 1$ and thus the intermediate value $\xi$ satisfies $\xi \in\left[-\pi^{*} L, 0\right] \subset[-1,0]$. Then $\mathrm{E}\left[L R_{1}\left(-\pi^{*} L\right)\right]=\frac{\gamma(1+\gamma)}{2}\left(\pi^{*}\right)^{2} \mathrm{E}\left[\frac{L^{3}}{(1+\xi)^{\gamma+2}}\right]>0$ and the upper bound $\pi^{*} \leq \frac{\tilde{\chi}}{\gamma \tilde{\sigma}^{2}}=\tilde{\pi}^{*}$ follows from (14). We also have $1+\xi \geq 1-\pi^{*} L>0$ and thus $\frac{1}{1+\xi} \leq \frac{1}{1-\pi^{*} L}$. Consequently,

$$
\mathrm{E}\left[L R_{1}\left(-\pi^{*} L\right)\right]=\frac{\gamma(1+\gamma)}{2}\left(\pi^{*}\right)^{2} \mathrm{E}\left[\frac{L^{3}}{(1+\xi)^{\gamma+2}}\right] \leq \frac{\gamma(1+\gamma)}{2}\left(\pi^{*}\right)^{2} C\left(\pi^{*}\right)
$$

Next, we use the upper bound $\tilde{\pi}^{*}=\frac{\tilde{\chi}}{\gamma \tilde{\sigma}^{2}}$ (adjusted to satisfy (3) if needed) and obtain $\mathrm{E}\left[L R_{1}\left(-\pi^{*} L\right)\right] \leq \frac{\gamma(1+\gamma)}{2}\left(\tilde{\pi}^{*}\right)^{2} C\left(\tilde{\pi}^{*}\right)$. Substituting into (14) yields $\pi^{*} \geq \frac{\tilde{\chi}}{\gamma \tilde{\sigma}^{2}}-\frac{(1+\gamma) \lambda}{2 \tilde{\sigma}^{2}}\left(\tilde{\pi}^{*}\right)^{2} C\left(\tilde{\pi}^{*}\right)$,

\footnotetext{
${ }^{19}$ See Proposition 12.5 in Cont and Tankov (2004) or Friedman (1975).

${ }^{20}$ See Protter (2005), pp. 311ff.
} 
which is the asserted lower bound.

Proof of Proposition 2.3. First, we derive a sharper upper bound on $\pi^{*}$. The first-order condition (14) is equivalent to

$$
\pi^{*}=\frac{\tilde{\chi}}{\gamma \tilde{\sigma}^{2}}-\frac{\lambda(1+\gamma)}{2 \tilde{\sigma}^{2}}\left(\pi^{*}\right)^{2} \mathrm{E}\left[\frac{L^{3}}{(1+\xi)^{\gamma+2}}\right]
$$

From (12) and the admissibility conditions (3) and (4), it follows that $-1<\xi \leq 0$. Therefore, $\frac{1}{1+\xi} \geq 1$ and thus $\mathrm{E}\left[\frac{L^{3}}{(1+\xi)^{\gamma+2}}\right] \geq \mathrm{E}\left[L^{3}\right]=\bar{L}_{3}$. Plugging this bound into (34) then gives $\pi^{*} \leq \frac{\tilde{\chi}}{\gamma \tilde{\sigma}^{2}}-A_{u}\left(\pi^{*}\right)^{2}$ with $A_{u}>0$. The solution of this quadratic inequality yields a lower and an upper bound on $\pi^{*}$. The lower bound is negative and is thus redundant due to (4). The upper bound is given by $\pi_{u}$. Next, we derive a non-trivial lower bound. From (33), we have that $\pi^{*} \geq \frac{\tilde{\chi}}{\gamma \tilde{\sigma}^{2}}-\frac{\lambda(1+\gamma)}{2 \tilde{\sigma}^{2}} C\left(\pi^{*}\right)\left(\pi^{*}\right)^{2}$. Since $C(\pi)$ is increasing, we use $\pi_{u}$ as an upper bound in $C\left(\pi^{*}\right)$ to obtain the quadratic inequality $\pi^{*} \geq \frac{\tilde{\chi}}{\gamma \tilde{\sigma}^{2}}-A_{l}\left(\pi^{*}\right)^{2}$. This yields the lower bound $\pi_{l}$, whereas the corresponding upper bound is negative and thus obsolete.

Proof of Proposition 2.4. The first-order condition of the problem is stated in (13). Condition (15) implies that the Merton-Breeden term is positive. The myopic demand in (16) thus gives a lower bound for $\pi^{*}$. Furthermore, jumps always decrease the stock price by (12). If we set $L=0$, the stock in this market is more attractive than the stock with jumps in the original market. Hence, the strategy given in (17) is an upper bound on the optimal strategy $\pi^{*}$.

Proof of Proposition 2.5. By the HJB equation (6), the function $g$ in (5) satisfies

$$
\begin{aligned}
0= & g_{t}+(1-\gamma)\left(r+\pi^{*} \chi-0.5 \gamma\left(\pi^{*}\right)^{2} \sigma^{2}\right) g+\left[\alpha+(1-\gamma) \pi^{*} \sigma \beta \rho\right] g_{y}+0.5 \beta^{2} g_{y y} \\
& +\lambda\left\{\mathrm{E}\left[\left(1-\pi^{*} L\right)^{1-\gamma}\right]-1\right\} g
\end{aligned}
$$


Differentiating (35) with respect to the state variable $y$ gives

$$
\begin{aligned}
0= & g_{t y}+(1-\gamma)\left(\pi_{y}^{*} \chi+\pi^{*} \chi_{y}-\gamma \pi^{*} \pi_{y}^{*} \sigma^{2}-0.5 \gamma\left(\pi^{*}\right)^{2}\left(\sigma^{2}\right)_{y}\right) g+(1-\gamma)\left(r+\pi^{*} \chi-0.5 \gamma\left(\pi^{*}\right)^{2} \sigma^{2}\right) g_{y} \\
& +\left[\alpha_{y}+(1-\gamma)\left(\pi_{y}^{*} \sigma \beta+\pi^{*}(\sigma \beta)_{y}\right) \rho\right] g_{y}+\left[\alpha+(1-\gamma) \pi^{*} \sigma \beta \rho\right] g_{y y}+0.5\left(\beta^{2}\right)_{y} g_{y y}+0.5 \beta^{2} g_{y y y} \\
& +\lambda_{y}\left\{\mathrm{E}\left[\left(1-\pi^{*} L\right)^{1-\gamma}\right]-1\right\} g-\lambda(1-\gamma) \pi_{y}^{*} \mathrm{E}\left[L\left(1-\pi^{*} L\right)^{-\gamma}\right] g+\lambda\left\{\mathrm{E}\left[\left(1-\pi^{*} L\right)^{1-\gamma}\right]-1\right\} g_{y}
\end{aligned}
$$

Sorting terms and denoting the coefficient of $g$ by $\check{D}$ shows that $\check{D}$ is equal to (18). Applying the Feynman-Kac theorem gives $g_{y}(t, y)=\int_{t}^{T} \check{\mathrm{E}}^{t, y}\left[e^{-\int_{t}^{s} \check{r}_{u} d u} \check{D}_{s} g\left(s, Y_{s}\right)\right] d s$, where $-\check{r}=(1-$ $\gamma)\left(r+\pi^{*} \chi-0.5 \gamma\left(\pi^{*}\right)^{2} \sigma^{2}\right)+\left[\alpha_{y}+(1-\gamma)\left(\pi_{y}^{*} \sigma \beta+\pi_{y}^{*}(\sigma \beta)_{y}\right) \rho\right]+\lambda\left\{\mathrm{E}\left[\left(1-\pi^{*} L\right)^{1-\gamma}\right]-1\right\}$ and the expectation $\check{\mathrm{E}}[\cdot]$ is taken under a measure under which $Y$ has drift $\alpha+(1-\gamma) \pi^{*} \sigma \beta \rho+0.5\left(\beta^{2}\right)_{y}$. Since $g>0$, the derivative $g_{y}$ is positive (negative) if $\check{D}$ is positive (negative).

Proof of Theorem 2.6. (i) The functions $g \equiv g(\cdot, \cdot ; \pi)$ and $\tilde{g} \equiv \tilde{g}(\cdot, \cdot ; \tilde{\pi})$ in (19) satisfy

$$
\begin{aligned}
0= & g_{t}+(1-\gamma)\left(r+\pi \chi-0.5 \gamma \pi^{2} \sigma^{2}\right) g+[\alpha+(1-\gamma) \pi \sigma \beta \rho] g_{y}+0.5 \beta^{2} g_{y y} \\
& +\lambda\left\{\mathrm{E}\left[(1-\pi L)^{1-\gamma}\right]-1\right\} g, \\
0= & \tilde{g}_{t}+(1-\gamma)\left(r+\tilde{\pi} \chi-0.5 \gamma \tilde{\pi}^{2} \sigma^{2}\right) \tilde{g}+[\alpha+(1-\gamma) \tilde{\pi} \sigma \beta \rho] \tilde{g}_{y}+0.5 \beta^{2} \tilde{g}_{y y} \\
& -\lambda \tilde{\pi}(1-\gamma)\left(\bar{L}_{1}+0.5 \gamma \tilde{\pi} \bar{L}_{2}\right) \tilde{g} .
\end{aligned}
$$

We set $D \equiv g-\tilde{g}$ and define the process $\Delta$ such that $\tilde{\pi}=\pi(1+\Delta)$. Furthermore, the Taylor series of $(1-L \pi)^{1-\gamma}$ converges, since this is in fact a binomial series and $1-\pi L<0$. Its second-order expansion reads $(1-\pi L)^{1-\gamma}=1-(1-\gamma) \pi L-0.5 \gamma(1-\gamma) \pi^{2} L^{2}+R_{2}(-\pi L)$, where $R_{2}(-\pi L) \equiv \frac{\gamma\left(1-\gamma^{2}\right)}{6} \frac{1}{(1+\xi)^{\gamma+2}}(-\pi L)^{3}$ with a suitable $\xi \in[-\pi L, 0]$, denotes the remainder term. Subtracting (37) from (36) and rearranging terms yields

$$
0=D_{t}-\hat{r} D+\hat{\alpha} D_{y}+0.5 \beta^{2} D_{y y}+\hat{D}
$$


where $-\hat{r} \equiv(1-\gamma)\left(r+\pi \chi-0.5 \gamma \pi^{2} \sigma^{2}\right)+\lambda\left[\mathrm{E}\left[(1-\pi L)^{1-\gamma}\right]-1\right], \hat{\alpha} \equiv \alpha+(1-\gamma) \pi \sigma \beta \rho$, and

$$
\hat{D}=\left\{\lambda \mathrm{E}\left[R_{2}(-\pi L)\right]-(1-\gamma) \Delta \pi\left[\chi-\lambda \bar{L}_{1}+\sigma \beta \rho \tilde{g}_{y} / \tilde{g}-0.5 \gamma(2+\Delta) \pi\left(\sigma^{2}+\lambda \bar{L}_{2}\right)\right]\right\} \tilde{g} .
$$

(ii) follows because $\tilde{\pi}^{*}$ satisfies the first-order condition $\chi-\lambda \bar{L}_{1}-\gamma \tilde{\pi}^{*}\left(\sigma^{2}+\lambda \bar{L}_{2}\right)+\sigma \beta \rho \tilde{g}_{y} / \tilde{g}=0$. (iii) follows from Proposition 2.1.

Proof of Proposition 3.2. (i) The first-order condition of (6) reads

$$
\gamma \sigma^{2} \pi=\chi+\sigma \beta \rho \frac{g_{y}}{g}-\lambda \mathrm{E}\left[L(1-\pi L)^{-\gamma}\right]
$$

Using (22) the local variance cancels and we obtain $\gamma \pi=\bar{\chi}+\bar{\beta} \rho \frac{g_{y}}{g}-\bar{\lambda} \mathrm{E}\left[L(1-\pi L)^{-\gamma}\right]$. Equation (27) follows for $\rho=0$.

(ii) Using the affine model (22) in (36) with $\rho=0$ yields the following PDE for $g_{0}(t, y ; \pi)$ :

$$
0=g_{0, t}+(1-\gamma) r g_{0}+\theta g_{0, y}-\kappa y g_{0, y}+0.5 \bar{\beta}^{2} y g_{0, y y}+C_{0} y g_{0}
$$

with $C_{0} \equiv(1-\gamma)\left(\pi \bar{\chi}-\frac{1}{2} \gamma \pi^{2}+\frac{\bar{\lambda}}{1-\gamma}\left\{\mathrm{E}\left[(1-\pi L)^{1-\gamma}\right]-1\right\}\right)$ as $J_{0}(t, x, y)=\frac{1}{1-\gamma} x^{1-\gamma} g_{0}(t, x, \pi)$ is the indirect utility if we use the optimal strategy $\pi=\pi^{*}$. It is straightforward to check that $g_{0}(t, y ; \pi)$ given in $(28)$ solves $(40)$.

(iii) follows from (22) and $\rho=0$ in (39).

(iv) follows from the definition of $\ell$ in (20). 
Figure 1: Investment strategies for the affine model (22) with constant jump size for different risk aversion parameters $\gamma$. The correlation parameter $\rho$ is set to zero and thus the optimal strategy and the bounds are constant over time. The solid line is the optimal strategy. The dashed lines are the lower and upper bounds I (Proposition 2.2). The dotted lines are the bounds II (Proposition 2.3). Note that the upper bound I is the approximating strategy.

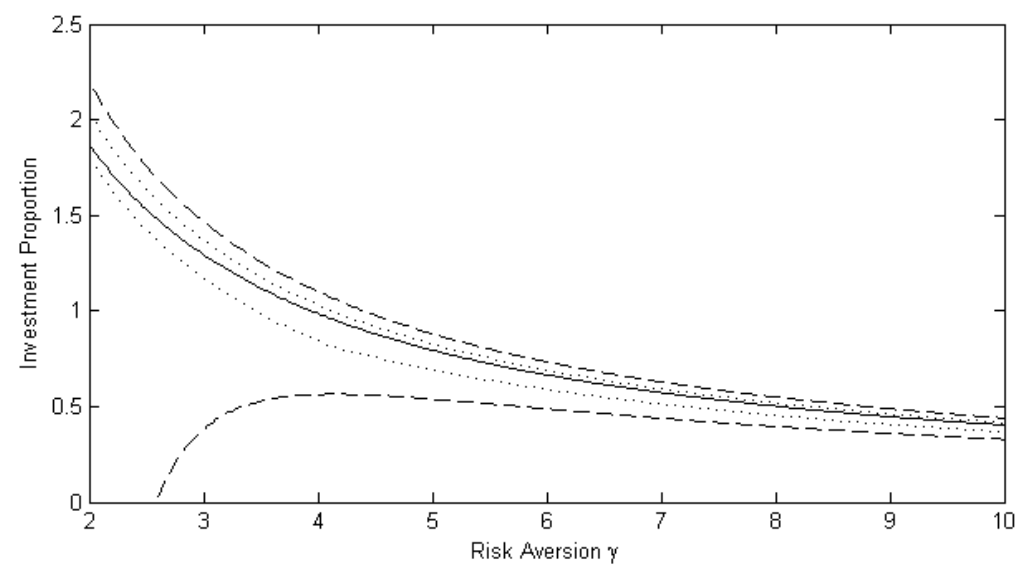

Figure 2: Investment strategies for the affine model (22) with beta-distributed jump size for different risk aversion parameters $\gamma$. The correlation parameter $\rho$ is set to zero and thus the optimal strategy and the bounds are constant over time. The solid line is the optimal strategy. The dashed lines are the lower and upper bounds I (Proposition 2.2). The dotted lines are the bounds II (Proposition 2.3). The investment cannot be higher than 1 due to (3). Note that the upper bound I is the approximating strategy.

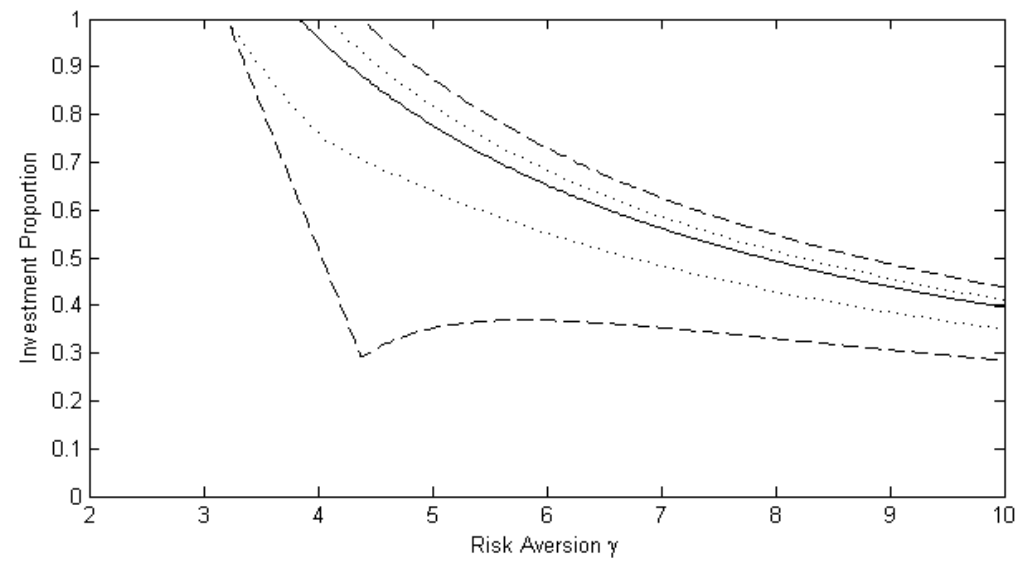


Figure 3: Investment strategies for the affine model (22) with log-normally distributed jump size for different risk aversion parameters $\gamma$. The correlation parameter $\rho$ is set to zero and thus the optimal strategy and the bounds are constant over time. The solid line is the optimal strategy. The dashed lines are the lower and upper bounds I (Proposition 2.2). The dotted lines are the bounds II (Proposition 2.3). The investment cannot be higher than 1 due to (3). Note that the upper bound I is the approximating strategy.

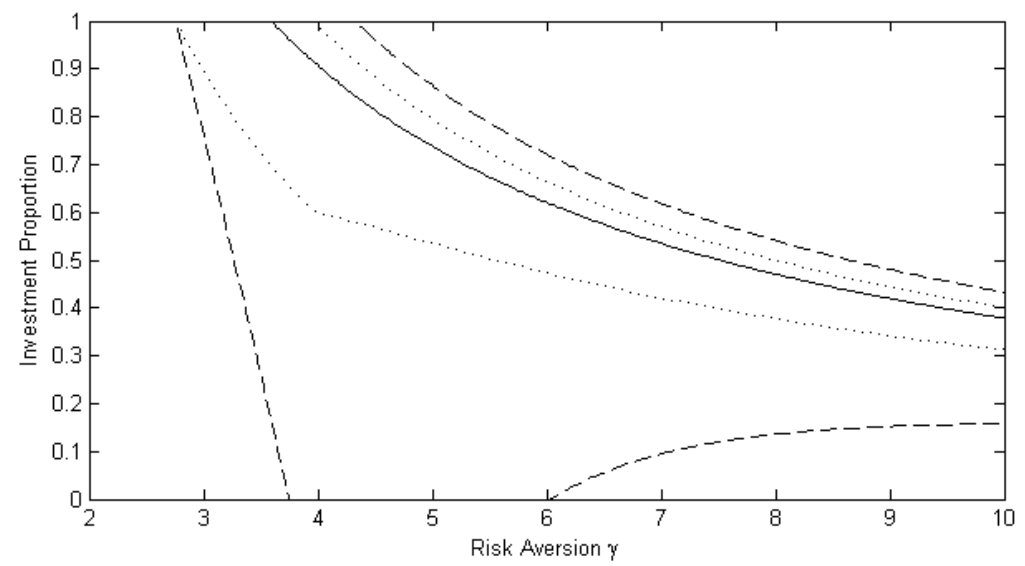

Figure 4: Lower bounds III on optimal investment $\pi_{t}^{*}$ implied by Proposition 2.4 for the affine model (22) for different risk aversion parameters $\gamma$. The remaining parameters are from (30). The black lines depict the lower bounds and the blue lines show the corresponding optimal strategies for $t=0$. The solid lines are for a deterministic jump size, the dashed lines for a beta-distributed jump size and the dotted lines for a log-normally distributed jump size. The upper bounds are large and thus omitted.

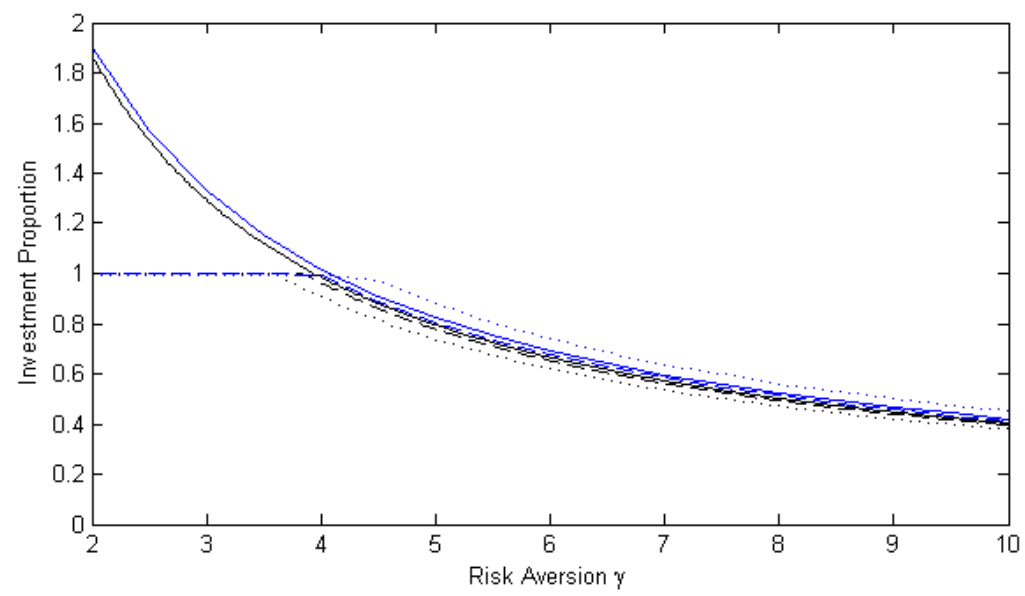


Figure 5: Relative wealth equivalent losses in the affine model (22) for different risk aversion parameters $\gamma$. The correlation is set to $\rho=0$, the remaining parameters are from (30). The solid line uses a deterministic jump size, the dashed line beta-distributed jump size and the dotted line log-normally distributed jump size.

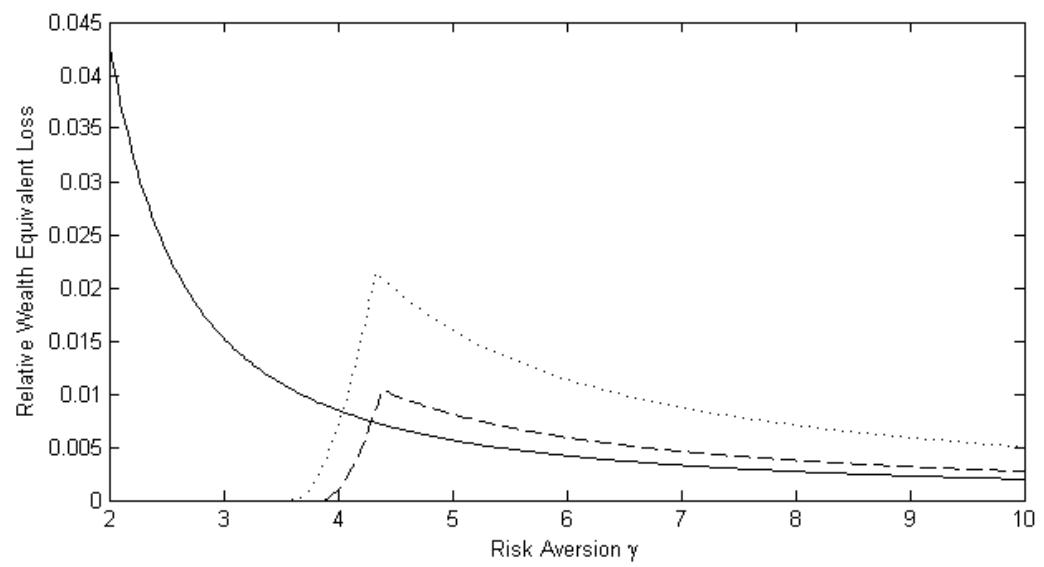

Table 1: RWELs for various risk aversions $\gamma$ and different jump specifications in the affine model (22). This table reports the RWELs that an investor using the optimal strategy would sacrifice in order to have the same indirect utility as an investor using the approximating strategy. The indirect utilities of the optimal strategy and approximating strategy in the true market are obtained using Proposition 3.1. Subsection 3.1 describes the three jump specifications. The reported values are in percentages.

\begin{tabular}{c|ccc}
\hline \hline Risk Aversion & \multicolumn{3}{|c}{ RWEL } \\
$\gamma$ & Constant & Beta & Log-normal \\
\hline 2.0 & 5.45 & 0.00 & 0.00 \\
3.0 & 2.06 & 0.00 & 0.00 \\
4.0 & 1.18 & 0.01 & 0.00 \\
5.0 & 0.80 & 1.16 & 0.73 \\
6.0 & 0.60 & 0.85 & 0.53 \\
7.0 & 0.48 & 0.67 & 0.41 \\
8.0 & 0.40 & 0.55 & 0.33 \\
9.0 & 0.34 & 0.47 & 0.28 \\
10.0 & 0.29 & 0.40 & 0.24 \\
\hline
\end{tabular}


Table 2: RWELs for different expected jump sizes $L$ and risk aversions $\gamma$. This table reports the RWELs that an investor using the optimal strategy would sacrifice in order to have the same indirect utility as an investor using the approximating strategy. The indirect utilities of the optimal strategy and approximating strategy in the true market are obtained using Proposition 3.1. When we vary the constant (expected) jump size, we adjust the parameters such that the expected excess return on stock remains the same as in the benchmark calibration (30). This procedure is described in Subsection 3.4. The quoted values are in percentages.

\begin{tabular}{c|rrrrrr}
\hline \hline Risk Aversion & \multicolumn{6}{|c}{ Constant Jump Size } \\
$\gamma$ & 0.05 & 0.10 & 0.15 & 0.20 & 0.25 & 0.30 \\
\hline 2 & 0.00 & 0.01 & 0.21 & 1.41 & 5.45 & 14.38 \\
3 & 0.00 & 0.01 & 0.09 & 0.57 & 2.06 & 5.20 \\
4 & 0.00 & 0.00 & 0.06 & 0.34 & 1.18 & 2.93 \\
5 & 0.00 & 0.00 & 0.04 & 0.23 & 0.80 & 1.98 \\
6 & 0.00 & 0.00 & 0.03 & 0.18 & 0.60 & 1.47 \\
7 & 0.00 & 0.00 & 0.03 & 0.14 & 0.48 & 1.17 \\
8 & 0.00 & 0.00 & 0.02 & 0.12 & 0.40 & 0.96 \\
9 & 0.00 & 0.00 & 0.02 & 0.10 & 0.34 & 0.82 \\
10 & 0.00 & 0.00 & 0.02 & 0.09 & 0.29 & 0.71 \\
\hline \hline Risk Aversion & \multicolumn{5}{|c}{ Expected Stock Jump Size (Beta) } \\
$\gamma$ & 0.05 & 0.10 & 0.15 & 0.20 & 0.25 & 0.30 \\
\hline 2 & 0.00 & 0.00 & 0.00 & 0.00 & 0.00 & 0.00 \\
3 & 0.00 & 0.00 & 0.00 & 0.00 & 0.00 & 0.00 \\
4 & 0.00 & 0.00 & 0.00 & 0.00 & 0.01 & 1.14 \\
5 & 0.00 & 0.01 & 0.10 & 0.40 & 1.16 & 2.55 \\
6 & 0.00 & 0.01 & 0.07 & 0.30 & 0.85 & 1.88 \\
7 & 0.00 & 0.01 & 0.06 & 0.23 & 0.67 & 1.48 \\
8 & 0.00 & 0.01 & 0.05 & 0.19 & 0.55 & 1.21 \\
9 & 0.00 & 0.01 & 0.04 & 0.16 & 0.47 & 1.02 \\
10 & 0.00 & 0.01 & 0.04 & 0.14 & 0.40 & 0.88 \\
\hline \hline Risk Aversion & Expected Stock Jump Size (Log-normal) \\
$\gamma$ & 0.05 & 0.10 & 0.15 & 0.20 & 0.25 & 0.30 \\
\hline 2 & 0.00 & 0.00 & 0.00 & 0.00 & 0.00 & 0.00 \\
3 & 0.00 & 0.00 & 0.00 & 0.00 & 0.00 & 0.00 \\
4 & 0.00 & 0.00 & 0.00 & 0.00 & 0.00 & 0.06 \\
5 & 0.00 & 0.00 & 0.00 & 0.12 & 0.72 & 2.03 \\
6 & 0.00 & 0.00 & 0.02 & 0.13 & 0.53 & 1.47 \\
7 & 0.00 & 0.00 & 0.02 & 0.10 & 0.41 & 1.14 \\
8 & 0.00 & 0.00 & 0.01 & 0.08 & 0.33 & 0.92 \\
9 & 0.00 & 0.00 & 0.01 & 0.07 & 0.28 & 0.78 \\
10 & 0.00 & 0.00 & 0.01 & 0.06 & 0.24 & 0.67 \\
\hline \hline
\end{tabular}


Table 3: RWELs for different jump intensity parameters $\bar{\lambda}$ and risk aversions $\gamma$. This table reports the RWELs that an investor using the optimal strategy would sacrifice in order to have the same indirect utility as an investor using the approximating strategy. The indirect utilities of the optimal strategy and approximating strategy in the true market are obtained using Proposition 3.1. When we vary the jump intensity parameter, we adjust the parameters such that the expected excess return on stock remains the same as in the benchmark calibration (30). This procedure is described in Subsection 3.4. For the constant jump size we use $L=0.25=$ const. For the beta-distributed jump size we use $\mathrm{E}(L)=0.25$ and $\operatorname{Var}(L)=0.05^{2}$, for the log-normally distributed jump size we use $\mathrm{E}(L)=0.25$ and $\operatorname{Var}(L)=0.1^{2}$. The quoted values are in percentages.

\begin{tabular}{|c|c|c|c|c|c|c|c|c|c|}
\hline Risk Aversion & \multicolumn{9}{|c|}{ Jump Intensity Parameter $\lambda$ (Constant) } \\
\hline$\gamma$ & 0.1 & 0.5 & 1.0 & 1.5 & 2.0 & 2.5 & 3.0 & 3.5 & 4.0 \\
\hline 2 & 0.08 & 1.20 & 2.97 & 4.55 & 5.82 & 6.80 & 7.53 & 8.05 & 8.41 \\
\hline 3 & 0.02 & 0.36 & 1.01 & 1.66 & 2.23 & 2.71 & 3.10 & 3.40 & 3.63 \\
\hline 4 & 01 & 0.19 & 0.55 & 0.94 & 1.29 & 1.59 & 1.83 & 2.03 & 2.19 \\
\hline 5 & 0.01 & 0.13 & 0.37 & 0.63 & 0.88 & 1.09 & 1.27 & 1.42 & 1.53 \\
\hline 6 & 00 & 0.09 & 0.27 & 0.47 & 0.66 & 0.82 & 0.96 & 1.08 & 1.17 \\
\hline 7 & 00 & 0.07 & 0.22 & 0.37 & 0.53 & 0.66 & 0.77 & 0.86 & 0.94 \\
\hline 8 & 00 & 0.06 & 0.18 & 0.31 & 0.43 & 0.55 & 0.64 & 0.72 & 0.78 \\
\hline 9 & 00 & 0.05 & 0.15 & 0.26 & 0.37 & 0.47 & 0.55 & 0.61 & 0.67 \\
\hline 10 & 0.00 & 0.04 & 0.13 & 0.23 & 0.32 & 0.41 & 0.48 & 0.54 & 0.58 \\
\hline Risk Aversion & \multicolumn{9}{|c|}{ Jump Intensity Parameter $\bar{\lambda}$ (Beta) } \\
\hline$\gamma$ & 0.1 & 0.5 & 1.0 & 1.5 & 2.0 & 2.5 & 3.0 & 3.5 & 4.0 \\
\hline 2 & 0.00 & 0.00 & 0.00 & 0.00 & 0.00 & 0.00 & 0.00 & 0.00 & 0.00 \\
\hline 3 & 00 & 0.00 & 0.00 & 0.00 & 0.00 & 0.00 & 0.00 & 0.00 & 0.00 \\
\hline 4 & 0.00 & 0.00 & 0.00 & 0.00 & 0.05 & 0.37 & 0.95 & 1.73 & 2.66 \\
\hline 5 & 0.00 & 0.20 & 0.56 & 0.93 & 1.26 & 1.53 & 1.74 & 1.91 & 2.04 \\
\hline 6 & 0.01 & 0.14 & 0.41 & 0.68 & 0.93 & 1.14 & 1.31 & 1.44 & 1.54 \\
\hline 7 & 0.01 & 0.11 & 0.32 & 0.53 & 0.73 & 0.90 & 1.04 & 1.15 & 1.23 \\
\hline 8 & 0.00 & 0.09 & 0.26 & 0.44 & 0.60 & 0.74 & 0.86 & 0.95 & 1.02 \\
\hline 9 & 0.00 & 0.07 & 0.22 & 0.37 & 0.51 & 0.63 & 0.73 & 0.81 & 0.87 \\
\hline 10 & 0.00 & 0.06 & 0.19 & 0.32 & 0.44 & 0.55 & 0.63 & 0.70 & 0.76 \\
\hline Risk Aversion & \multicolumn{9}{|c|}{ Jump Intensity Parameter $\lambda$ (Log-normal) } \\
\hline$\gamma$ & 0.1 & 0.5 & 1.0 & 1.5 & 2.0 & 2.5 & 3.0 & 3.5 & 4.0 \\
\hline 2 & 0.00 & 0.00 & 0.00 & 0.00 & 0.00 & 0.00 & 0.00 & 0.00 & 0.00 \\
\hline 3 & 0.00 & 0.00 & 0.00 & 0.00 & 0.00 & 0.00 & 0.00 & 0.00 & 0.00 \\
\hline 4 & 0.00 & 0.00 & 0.00 & 0.00 & 0.00 & 0.00 & 0.00 & 0.01 & 0.10 \\
\hline 5 & 0.00 & 0.03 & 0.29 & 0.54 & 0.81 & 1.08 & 1.34 & 1.59 & 1.83 \\
\hline 6 & 0.00 & 0.06 & 0.21 & 0.39 & 0.59 & 0.79 & 0.99 & 1.18 & 1.35 \\
\hline 7 & 0.00 & 0.05 & 0.16 & 0.30 & 0.46 & 0.62 & 0.78 & 0.93 & 1.07 \\
\hline 8 & .00 & 0.04 & 0.13 & 0.25 & 0.37 & 0.51 & 0.64 & 0.76 & 0.88 \\
\hline 9 & 0.00 & 0.03 & 0.11 & 0.21 & 0.32 & 0.43 & 0.54 & 0.64 & 0.75 \\
\hline 10 & 0.00 & 0.03 & 0.09 & 0.18 & 0.27 & 0.37 & 0.46 & 0.56 & 0.65 \\
\hline
\end{tabular}


Table 4: RWELs for different jump parametrizations, but constant average impact of jumps. This table reports the RWELs that an investor using the optimal strategy would sacrifice in order to have the same indirect utility as an investor using the approximating strategy. The indirect utilities of the optimal strategy and approximating strategy in the true market are obtained using Proposition 3.1. We vary the jump intensity and the jump size such that the average impact of jumps stays constant, i.e. we fix the product $\bar{\lambda} \bar{L}_{1}$ to its value in the benchmark calibration (30). The quoted values are in percentages.

\begin{tabular}{c|rrrrrr}
\hline \hline Risk Aversion & \multicolumn{6}{|c}{ Constant Jump Size } \\
$\gamma$ & 0.05 & 0.1 & 0.15 & 0.2 & 0.25 & 0.3 \\
\hline 2.0 & 0.00 & 0.07 & 0.47 & 1.84 & 5.45 & 13.35 \\
3.0 & 0.00 & 0.04 & 0.21 & 0.76 & 2.06 & 4.60 \\
4.0 & 0.00 & 0.02 & 0.13 & 0.45 & 1.18 & 2.54 \\
5.0 & 0.00 & 0.02 & 0.09 & 0.32 & 0.80 & 1.70 \\
6.0 & 0.00 & 0.01 & 0.07 & 0.24 & 0.60 & 1.26 \\
7.0 & 0.00 & 0.01 & 0.06 & 0.19 & 0.48 & 1.00 \\
8.0 & 0.00 & 0.01 & 0.05 & 0.16 & 0.40 & 0.82 \\
9.0 & 0.00 & 0.01 & 0.04 & 0.14 & 0.34 & 0.69 \\
10.0 & 0.00 & 0.01 & 0.04 & 0.12 & 0.29 & 0.60 \\
\hline \hline Risk Aversion & \multicolumn{5}{|c}{ Expected Jump Size $($ Beta) } \\
$\gamma$ & 0.05 & 0.1 & 0.15 & 0.2 & 0.25 & 0.3 \\
\hline 2.0 & 0.00 & 0.00 & 0.00 & 0.00 & 0.00 & 0.00 \\
3.0 & 0.00 & 0.00 & 0.00 & 0.00 & 0.00 & 0.00 \\
4.0 & 0.00 & 0.00 & 0.00 & 0.00 & 0.01 & 0.50 \\
5.0 & 0.06 & 0.08 & 0.21 & 0.53 & 1.16 & 2.23 \\
6.0 & 0.05 & 0.06 & 0.16 & 0.40 & 0.85 & 1.63 \\
7.0 & 0.04 & 0.05 & 0.13 & 0.31 & 0.67 & 1.28 \\
8.0 & 0.03 & 0.04 & 0.11 & 0.26 & 0.55 & 1.04 \\
9.0 & 0.03 & 0.04 & 0.09 & 0.22 & 0.47 & 0.88 \\
10.0 & 0.02 & 0.03 & 0.08 & 0.19 & 0.40 & 0.76 \\
\hline \hline Risk Aversion & Expected Jump & Size (Log-normal) \\
$\gamma$ & 0.05 & 0.1 & 0.15 & 0.2 & 0.25 & 0.3 \\
\hline 2.0 & 0.00 & 0.00 & 0.00 & 0.00 & 0.00 & 0.00 \\
3.0 & 0.00 & 0.00 & 0.00 & 0.00 & 0.00 & 0.00 \\
4.0 & 0.00 & 0.00 & 0.00 & 0.00 & 0.00 & 0.00 \\
5.0 & 0.00 & 0.00 & 0.00 & 0.23 & 0.72 & 1.68 \\
6.0 & 0.01 & 0.01 & 0.05 & 0.19 & 0.53 & 1.20 \\
7.0 & 0.01 & 0.01 & 0.04 & 0.15 & 0.41 & 0.93 \\
8.0 & 0.00 & 0.00 & 0.03 & 0.12 & 0.33 & 0.75 \\
9.0 & 0.00 & 0.00 & 0.03 & 0.10 & 0.28 & 0.63 \\
10.0 & 0.00 & 0.00 & 0.02 & 0.09 & 0.24 & 0.54 \\
\hline \hline
\end{tabular}


Table 5: RWELs of the alternative approximating strategy for different jump intensity parameters $\bar{\lambda}$ and risk aversions $\gamma$. This table reports the RWELs that an investor using the optimal strategy would sacrifice in order to have the same indirect utility as an investor using the alternative approximating strategy of Section 3.5. When we vary the jump intensity parameter, we adjust the parameters such that the expected excess return on stock remains the same as in the benchmark calibration (30). For the constant jump size we use $L=0.25=$ const. For the beta-distributed jump size we use $\mathrm{E}(L)=0.25$ and $\operatorname{Var}(L)=0.05^{2}$, for the log-normally distributed jump size we use $\mathrm{E}(L)=0.25$ and $\operatorname{Var}(L)=0.1^{2}$. The quoted values are in percentages.

\begin{tabular}{|c|c|c|c|c|c|c|c|c|c|}
\hline Risk Aversion & \multicolumn{9}{|c|}{ Jump Intensity Parameter $\bar{\lambda}$ (Constant) } \\
\hline$\gamma$ & 0.1 & 0.5 & 1.0 & 1.5 & 2.0 & 2.5 & 3.0 & 3.5 & 4.0 \\
\hline 2 & 0.00 & 0.01 & 0.02 & 0.03 & 0.03 & 0.03 & 0.03 & 0.03 & 0.03 \\
\hline 3 & 0.00 & 0.01 & 0.01 & 0.02 & 0.02 & 0.02 & 0.02 & 0.02 & 0.02 \\
\hline 4 & 0.00 & 0.00 & 0.01 & 0.01 & 0.01 & 0.02 & 0.02 & 0.02 & 0.02 \\
\hline 5 & 0.00 & 0.00 & 0.01 & 0.01 & 0.01 & 0.01 & 0.01 & 0.01 & 0.01 \\
\hline 6 & .00 & 0.00 & 0.00 & 0.01 & 0.01 & 0.01 & 0.01 & 0.01 & 0.01 \\
\hline 7 & 00 & 0.00 & 0.00 & 0.01 & 0.01 & 0.01 & 0.01 & 0.01 & 0.01 \\
\hline 8 & 0.00 & 0.00 & 0.00 & 0.01 & 0.01 & 0.01 & 0.01 & 0.01 & 0.01 \\
\hline 9 & 0.00 & 0.00 & 0.00 & 0.00 & 0.01 & 0.01 & 0.01 & 0.01 & 0.01 \\
\hline 10 & 0.00 & 0.00 & 0.00 & 0.00 & 0.01 & 0.01 & 0.01 & 0.01 & 0.01 \\
\hline Risk Aversion & \multicolumn{9}{|c|}{ "Jump Intensity Parameter $\lambda$ (Beta) } \\
\hline$\gamma$ & 0.1 & 0.5 & 1.0 & 1.5 & 2.0 & 2.5 & 3.0 & 3.5 & 4.0 \\
\hline 2 & 0.00 & 0.00 & 0.00 & 0.00 & 0.00 & 0.00 & 0.00 & 0.00 & 0.00 \\
\hline 3 & 0.00 & 0.00 & 0.00 & 0.00 & 0.00 & 0.00 & 0.00 & 0.00 & 0.00 \\
\hline 4 & 0.00 & 0.00 & 0.00 & 0.00 & 0.02 & 0.02 & 0.02 & 0.02 & 0.02 \\
\hline 5 & 0.00 & 0.00 & 0.01 & 0.01 & 0.02 & 0.02 & 0.02 & 0.02 & 0.02 \\
\hline 6 & 0.00 & 0.00 & 0.01 & 0.01 & 0.01 & 0.01 & 0.01 & 0.01 & 0.01 \\
\hline 7 & 0.00 & 0.00 & 0.01 & 0.01 & 0.01 & 0.01 & 0.01 & 0.01 & 0.01 \\
\hline 8 & 0.00 & 0.00 & 0.01 & 0.01 & 0.01 & 0.01 & 0.01 & 0.01 & 0.01 \\
\hline 9 & 0.00 & 0.00 & 0.00 & 0.01 & 0.01 & 0.01 & 0.01 & 0.01 & 0.01 \\
\hline 10 & 0.00 & 0.00 & 0.00 & 0.01 & 0.01 & 0.01 & 0.01 & 0.01 & 0.01 \\
\hline Risk Aversion & \multicolumn{9}{|c|}{ Jump Intensity Parameter $\lambda$ (Log-normal) } \\
\hline$\gamma$ & 0.1 & 0.5 & 1.0 & 1.5 & 2.0 & 2.5 & 3.0 & 3.5 & 4.0 \\
\hline 2 & 0.00 & 0.00 & 0.00 & 0.00 & 0.00 & 0.00 & 0.00 & 0.00 & 0.00 \\
\hline 3 & 0.00 & 0.00 & 0.00 & 0.00 & 0.00 & 0.00 & 0.00 & 0.00 & 0.00 \\
\hline 4 & 0.00 & 0.00 & 0.00 & 0.00 & 0.00 & 0.00 & 0.00 & 0.01 & 0.03 \\
\hline 5 & 0.00 & 0.00 & 0.01 & 0.01 & 0.01 & 0.02 & 0.02 & 0.02 & 0.02 \\
\hline 6 & 0.00 & 0.00 & 0.00 & 0.01 & 0.01 & 0.01 & 0.01 & 0.02 & 0.02 \\
\hline 7 & 0.00 & 0.00 & 0.00 & 0.01 & 0.01 & 0.01 & 0.01 & 0.01 & 0.01 \\
\hline 8 & 0.00 & 0.00 & 0.00 & 0.01 & 0.01 & 0.01 & 0.01 & 0.01 & 0.01 \\
\hline 9 & 0.00 & 0.00 & 0.00 & 0.00 & 0.01 & 0.01 & 0.01 & 0.01 & 0.01 \\
\hline 10 & 0.00 & 0.00 & 0.00 & 0.00 & 0.01 & 0.01 & 0.01 & 0.01 & 0.01 \\
\hline
\end{tabular}

